\title{
Existence and Smoothing Effect of Solutions for the Zakharov Equations
}

\author{
By \\ Tohru OzawA*† and Yoshio Tsutsumi**:
}

\section{$\S 1$. Introduction and Theorems}

In the present paper we consider the unique local solvability and the smoothing effect for the Zakharov equations:

$$
\begin{aligned}
& i \frac{\partial E}{\partial t}+\Delta E=n E, \quad t>0, \quad x \in \boldsymbol{R}^{N}, \\
& \frac{\partial^{2} n}{\partial t^{2}}-\Delta n=\Delta|E|^{2}, \quad t>0, \quad x \in \boldsymbol{R}^{N}, \\
& E(0, x)=E_{0}(x), \quad n(0, x)=n_{0}(x), \quad \frac{\partial}{\partial t} n(0, x)=n_{1}(0, x),
\end{aligned}
$$

where $E$ is a function from $\boldsymbol{R}_{t}^{+} \times \boldsymbol{R}_{x}^{N}$ to $\boldsymbol{C}^{N}, n$ is a function from $\boldsymbol{R}_{t}^{+} \times \boldsymbol{R}_{x}^{N}$ to $\boldsymbol{R}$ and $1 \leqq N \leqq 3$. (1.1)-(1.3) describe the long wave Langmuir turbulence in a plasma (see [20]). $E(t, x)$ denotes the slowly varying envelope of the highly oscillatory electric field and $n(t, x)$ denotes the deviation of the ion density from its equilibrium. When (1.2) depends on the ion sound speed $c$, that is, (1.2) is replaced by

$$
\frac{1}{c^{2}} \frac{\partial^{2} n}{\partial t^{2}}-\Delta n=\Delta|E|^{2}
$$

it is thought that (1.1) and (1.4) converge to

Communicated by S. Matsuura, September 17, 1990.

1991 Mathematics Subject Classifications: 35Q55

* Laboratoire de Physique Théorique et Hautes Energies Université de Paris XI Bâtiment 211, 91405 Orsay Cedex, France

*** Department of Mathemarics Nagoya University Chikusa-ku, Nagoya 464-01 Japan

$\dagger$ On leave of absence from Research Institute for Mathematical Sciences, Kyoto University, Kyoto 606, Japan.

Current Address: Department of Mathematics, Hokkaido University, Sapporo 060, Japan

\# Current Address: Department of Mathematical Sciences, University of Tokyo, Hongo, Tokyo 113, Japan 


$$
i \frac{\partial E}{\partial t}+\Delta E=n E, \quad n=-|E|^{2}
$$

as $c \rightarrow \infty$ (see [2], [17] and [20]). (1.5) is just the nonlinear Schrödinger equation and it is conjectured that the solutions of (1.1)-(1.2) and the solution of (1.5) have some common properties (see, e.g., [12, $\$ 1$. Introduction]). The Zakharov equations (1.1)-(1.3) have not yet been studied well, while the nonlinear Schrödinger equation (1.5) has extensively been studied (see, e.g., [3]-[9], [11], [15] and [18]).

In [17] C. Sulem and P.L. Sulem proved by using the Galerkin method that if $\left(E_{0}, n_{0}, n_{1}\right) \in H^{m} \oplus H^{m-1} \oplus\left(H^{m-2} \cap \dot{H}^{-1}\right), m \geqq 3$ and $1 \leqq N \leqq 3$, then (1.1)-(1.3) have the unique local solutions $(E, n) \in L^{\infty}\left(0, T ; H^{m}\right) \oplus L^{\infty}\left(0, T ; H^{m-1}\right)$ for some $T>0$. Here $H^{m}$ denotes the standard Sobolev space $H^{m}\left(\mathbb{R}^{N}\right) . \quad \dot{H}^{m}$ denotes the homogeneous Sobolev space consisting of all tempered distributions $u$ with $|\xi|^{m} \hat{u} \in L^{2} \equiv L^{2}\left(\boldsymbol{R}^{N}\right)$, where $\hat{u}$ is the Fourier transform of $u$. In [12] Schochet and Weinstein showed a similar result for $(1.1),(1.4)$ and $(1.3)$ by the different method, but the existence time $T$ of the local solutions does not depend on the parameter $c$ in [12] (see also [2]). In both [12] and [17], the assumption $n_{1} \in \dot{H}^{-1}$ is needed for the construction of the local solutions. This assumption is rather strong, because $S \subset \dot{H}^{-1}$ for $N=1,2$. For example, $e^{-|x|^{2}}$ is not in $\dot{H}^{-1}$ for $N=1,2$. Furthermore, the uniqueness of the solutions $(E(t)$, $n(t))$ for (1.1)-(1.3) is proved only in the class $H^{m} \oplus H^{m-1}, m \geqq 3$, in the previous results. In this paper we first show the unique local existence, result in $H^{2} \oplus H^{1}$ for (1.1)-(1.3). $\quad H^{2} \oplus H^{1}$ seems more natural than the class in the previous results, because the solutions in $H^{2} \oplus H^{1}$ are the so-called strong solutions.

We next investigate the smoothing effect of the solutions for (1.1)-(1.3). It is well known that the nonlinear Schrödinger equation (1.5) has the drastic smoothing effect (see, e.g., [4], [7]-[10] and [15]). In [7] and [8] it is proved that if $E(0)=E_{0} \in H^{1},|x|^{k} E_{0} \in L^{2}, k \geqq 1$ and $1 \leqq N \leqq 3$, then the solution $E(t)$ of (1.5) is in $H_{l o c}^{k} \equiv H_{l o c}^{k}\left(\boldsymbol{R}^{N}\right)$ for $t>0$ as long as $E(t)$ exists. In [4] and [15] the smoothing effect of different type for (1.5) is proved, that is, if $E(0)=E_{0} \in$ $H^{k}, k \geqq 1$ and $1 \leqq N \leqq 3$, then the solution $E(t)$ of (1.5) satisfies.

$$
\int_{0}^{T}\left\|\varphi(1-\Delta)^{k / 2+1 / 4} E(t)\right\|_{L^{2}}^{2} d t \leqq C
$$

for $\varphi \in C_{0}^{\infty}\left(\boldsymbol{R}^{N}\right)$ and $0<T<T_{\max }$, where $T_{\max }$ is the maximal existence time of $E(t)$. On the other hand, there seems to be no result concerning the smoothing property of (1.1)-(1.3). The Zakharov system consists of the Schrödinger equa- 
tion (1.1) and the wave equation (1.2), and we cannot expect the smoothing effect of the wave equation part. Accordingly, we cannot expect the drastic smoothing effect for (1.1)-(1.3) like the single nonlinear Schrödinger equation. Nevertheless, we can prove that the solution $E(t)$ of the Schrödinger part for (1.1)-(1.3) has some smoothing properties.

Before we state the main results in this paper, we define several function spaces. Let $W^{m, p}$ denote the Sobolev space

$$
W^{m, p}=\left\{f \in \mathcal{S}^{\prime} ;\|f\|_{W^{m, p}} \equiv\left\|(1-\Delta)^{m / 2} f\right\|_{\left.L^{p}<\infty\right\}}\right.
$$

for $m \in \boldsymbol{R}$ and $1<p<\infty$. We put $H^{m} \equiv W^{m, 2}$. Let $H^{m, s}$ denote the weighted Sobolev space

$$
H^{m, s}=\left\{f \in \mathcal{S}^{\prime} ;\|f\|_{H, s} \equiv\left\|\left(1+|x|^{2}\right)^{s / 2}(1-\Delta)^{m / 2} f\right\|_{L^{2}}<\infty\right\}
$$

for $m, s \in \boldsymbol{R}$. For a Banach space $X$ and $T>0$, we define $W^{m, p}(0, T ; X)$ by

$$
W^{m, p}(0, T ; X) \equiv\left\{f(t) \in L^{p}(0, T ; X) ;\left[\sum_{j=0}^{m} \int_{0}^{T}\left\|\frac{d^{j}}{d t^{j}} f(t)\right\|_{X}^{p} d t\right]^{1 / p}<\infty\right\},
$$

if $1 \leqq p<\infty$ and

$$
W^{m, \infty}(0, T ; X) \equiv\left\{f(t) \in L^{\infty}(0, T ; X) ; \sum_{j=0}^{m} \sup _{0 \leqq t \leqq T}\left\|\frac{d^{j}}{d t^{j}} f(t)\right\|_{X}<\infty\right\}
$$

if $p=\infty$.

The main results in this paper are the following.

Theorem 1.1. Assume that $1 \leqq N \leqq 3$.

(1) Let $\left(E_{0}, n_{0}, n_{1}\right) \in H^{2} \oplus H^{1} \oplus L^{2}$. Then for some $T>0$ there exist the unique strong solutions $(E(t), n(t))$ of (1.1)-(1.3) such that

$$
\begin{aligned}
& E \in \bigcap_{j=0}^{1} C^{j}\left([0, T] ; H^{2-2 j}\right), \\
& E \in \bigcap_{j=0}^{1} W^{j, 8 / N}\left(0, T ; W^{2-2 j, 4}\right), \\
& n \in \bigcap_{j=0}^{2} C^{j}\left([0, T] ; H^{1-j}\right),
\end{aligned}
$$

where $T$ depends only on $\left\|E_{0}\right\|_{H^{2}},\left\|n_{0}\right\|_{H^{1}},\left\|n_{1}\right\|_{L^{2}}$ and $N$.

(2) Let $m$ be an even integer with $m \geqq 4$. If $\left(E_{0}, n_{0}, n_{1}\right) \in H^{m} \oplus H^{m-1} \oplus H^{m-2}$, then the solutions $(E(t), n(t))$ of (1.1)-(1.3) given by Part (1) satisfy 


$$
\begin{aligned}
& E \in \bigcap_{j=0}^{m / 2} C^{j}\left([0, T] ; H^{m-2 j}\right), \\
& E \in \bigcap_{j=0}^{m / 2} W^{j, 8 / N}\left(0, T ; W^{m-2 j, 4}\right), \\
& n \in \bigcap_{j=0}^{3} C^{j}\left([0, T] ; H^{m-1-j}\right),
\end{aligned}
$$

and if $m \geqq 6$,

$$
n \in \bigcap_{j=4}^{m / 2+1} C^{j}\left([0, T] ; H^{m+2-2 j}\right)
$$

(3) Let $m$ be an odd integer with $m \geqq 3$. If $\left(E_{0}, n_{0}, n_{1}\right) \in H^{m} \oplus H^{m-1} \oplus H^{m-2}$, then the solutions $(E(t), n(t))$ of (1.1)-(1.3) given by Part (1) satisfy

$$
\begin{aligned}
& E \in \bigcap_{j=0}^{(u-1) / 2} C^{j}\left([0, T] ; H^{m-2 j}\right), \\
& E \in \bigcap_{j=0}^{(m-1) / 2} W^{j, 8 / N}\left(0, T ; W^{m-2 j, 4}\right), \\
& n \in \bigcap_{j=0}^{3} C^{j}\left([0, T] ; H^{m-1-j}\right),
\end{aligned}
$$

and if $m \geqq 7$,

$$
n \in \bigcap_{j=4}^{(m+1) / 2} C^{j}\left([0, T] ; H^{m+2-2 j}\right)
$$

Remark 1.1 (1) The solutions $(E(t), n(t))$ of (1.1)-(1.3) in Theorem 1.1 (1) satisfy (1.1) in the $L^{2}$ sense, while they satisfy (1.2) in the distribution sense. Therefore, the solutions in the class of Theorem 1.1(1) are called the strong solutions (for the weak solutions, see [12, Theorem 4] and [17, Theorem 1]).

(2) Theorem 1.1 (1) shows that the solutions of (1.1)-(1.3) are unique in the class of the strong solutions.

(3) In Theorem 1.1 we do not need the condition $n_{1} \in \dot{H}^{-1}$, which was always assumed in the previous papers [12] and [17].

(4) In Theorems 1.1(2) and (3) the existence time $T$ of the more regular solutions than the strong solutions is the same as that of the strong solutions. In the previous papers [12] and [17], $T$ depends on the higher order Sobolev norms of the initial data, when the solutions are regular. Theorems 1.1(2) and (3) imply that if $\left(E_{0}, n_{0}, n_{1}\right) \in \bigcap_{k=1}^{\infty} H^{k}$, then the solutions $(E, n) \in C^{\infty}\left([0, T] \times \mathbb{R}^{N}\right)$. (5) (1.7) (1.10) and (1.14) show that $E(t)$ has a smoothing property in a 
certain sense like the solution of the single nonlinear Schrödinger equation (see [6], [11], [16] and [19]).

(6) (1.12) and (1.16) imply that $\frac{\partial^{j} n}{\partial t^{j}}, j \geqq 3$, lose the regularity of Sobolev order 2 with respect to the spatial variables, each time we differentiate them in $t$. This may seem strange, since $n(t)$ is a solution of the wave equation. But (1.2) contains the solution $E(t)$ of the Schrödinger equation as the external force, which is why (1.12) and (1.16) occur.

Theorem 1.2. Let $m$ be an integer with $m \geqq 2$. Assume that $1 \leqq N \leqq 3$ and $\left(E_{0}, n_{0}, n_{1}\right) \in H^{m} \oplus H^{m-1} \oplus H^{m-2} . \quad$ Let $(E(t), n(t))$ and $T_{m a x}>0$ be the solutions of (1.1)-(1.3) given by Theorem 1.1 and their maximal existence time, respectively.

(1) Let $\varphi \in C_{0}^{\infty}\left(\boldsymbol{R}^{N}\right)$. Then $E(t)$ satisfies

$$
\varphi E \in L^{2}\left(0, T ; H^{m+1 / 2}\right)
$$

for any $T$ with $0<T<T_{\text {max }}$.

(2) In addition, let $m \geqq 4$. Put $k=1$ if $m \geqq 4$ and $k=1$ or 2 if $m \geqq 6$. If $E_{0} \in$ $H^{m, k}$, then

$$
E(t) \in H_{l o c}^{m+k}, \quad 0<t<T_{\max } .
$$

Remark 1.2. Theorem 1.2 shows the smoothing properties of the Zakharov equations (1.1)-(1.3). Part (1) is completely the same as in the case of the single nonlinear Schrödinger equation (see [4] and [15]). On the other hand, Part (2) is not so good as in the case of the single nonlinear Schrödinger equation (see [7]-[9]). This is because the Zakharov system contains the wave equation and it has the form such that the derivative loss occurs.

The difficulty of solving (1.1)-(1.3) is that when we use the standard iteration scheme, we meet with the loss of derivative, which comes from the second derivatives of $|E(t)|^{2}$ in (1.2). In the case of the single nonlinear Schrödinger equation the $L^{p}-L^{q}$ estimate and the Strichartz estimate play an important role (see [5], [6], [11] and [19]). However, in the previous papers [12] and [17] they are not used, because the loss of derivative prevents us from using them. In our proof of Theorem 1.1 we first transform (1.1)-(1.2) into the system which does not have the derivative loss. For that purpose, we apply the technique developed by Shibata and Y. Tsutsumi [13], which was used to solve the fully nonlinear wave equation. After that we apply the $L^{p}-L^{q}$ estimate and the Strichartz estimate to the resulting system, following Kato [11]. 
When we investigate the smoothing effect of (1.1)-(1.3), the derivative loss of (1.1)-(1.3) causes difficulty again. In addition, we can not expect the solution $n(t)$ of the wave equation part to have a smoothing property. However, we can derive the smoothing effect for the solution $E(t)$ of the Schrödinger equation part by using the smoothing effect peculiar to the Schrödinger equation (see [4], [7]-[10], [14] and [15]) and the difference between the Schrödinger equation and the wave equation. Especially in our proof of Theorem 1.2(2) the difference of the derivative in $t$ between the Schrödinger equation and the wave equation plays an important role.

Our plan in this paper is as follows. In Section 2 we prepare several lemmas needed for the proofs of Theorems 1.1 and 1.2. In Section 3 we give the proof of Theorem 1.1 and state some results concerning the existence of global solutionds for (1.1)-(1.3). In Section 4 we give the proof of Theorem 1.2.

Finally we conclude this section by giving several notations. Let $\left(\circ^{\circ},{ }^{\circ}\right)$ denote the scalar product in $L^{2}$. We abbreviate $\frac{\partial}{\partial t}$ and $\frac{\partial}{\partial x_{k}}, 1 \leqq k \leqq N$, to $\partial_{t}$ and $\partial_{k}, 1 \leqq k \leqq N$, respectively. Let $\alpha(N)=\infty$ if $N=1,2$ and $\alpha(N)=\frac{2 N}{N-2}$ if $N \geqq 3$. By $U(t)$ we denote the evolution operator of the free Schrödinger equation. We put $\omega=\sqrt{-\Delta}, J_{k}=x_{k}+2 i t \partial_{k}, 1 \leqq k \leqq N$, and $M(t)=e^{|x|^{2} / 4 i t}$. Let $\rho \in$ $C_{0}^{\infty}\left(\boldsymbol{R}^{N}\right)$ such that $\rho \geqq 0$ and $\|\rho\|_{L^{1}}=1$. We put $\rho_{\varepsilon}(x)=\varepsilon^{-N} \rho(x / \varepsilon)$ for $\varepsilon>0$. Let * denote the convolution with respect to the spatial variables. For $z \in \mathbb{C}$ we denote by $\bar{z}$ the complex conjugate of $z$. In the course of calculations below various positive constants are simply denoted by $C$.

\section{\$2. Lemmas}

In this section we summarize several lemmas needed for the proofs of Theorems 1.1 and 1.2.

We first state two lemmas concerning the space-time estimates of the evolution operator of the free Schrödinger equation.

Lemma 2.1. (i) Let $p$ and $q$ be two positive constants such that $2 \leqq p \leqq \infty$ and $1 / p+1 / q=1$. Then,

$$
\|U(t) v\|_{L^{p}} \leqq(4 \pi|t|)^{-N / 2+N / p}\|v\|_{L^{q}}, \quad t \neq 0 .
$$

(ii) Let $q$ and $r$ be two positive constants such that $2 \leqq q<\alpha(N)$ and $(N / 2-$ $N / q) r=2$. Then, there exists a positive constant $K_{1}$ depending only $N$ and $q$ 
such that

$$
\|U(\cdot) u\|_{L^{r}\left(\boldsymbol{R} ; L^{q}\right)} \leqq K_{1}\|v\|_{L^{2}}, \quad v \in L^{2} .
$$

(2.1) in Lemma 2.1(i) is the well known $L^{p}-L^{q}$ estimate and (2.2) in Lemma 2.1(ii) is the Strichartz estimate. For the proof of Lemma 2.1, see [5, Lemma 1.2], [6, Proposition 4.4] and [16, Corollary 1 in \$3].

Lemma 2.2. Let $q, r, q^{\prime}$ and $r^{\prime}$ be four positive constants such that $1 \leqq q^{\prime}, r^{\prime}$ $\leqq 2,1 / q+1 / q^{\prime}=1,1 / r+1 / r^{\prime}=1,2 \leqq q<\alpha(N)$ and $(N / 2-N / q) r=2$. Let $I$ be any interval in $\boldsymbol{R}$. There exists a positive constant $K_{2}$ depending only on $N$ and $q^{\prime}$ such that

$$
\left\|\int_{0}^{t} U(t-s) f(s) d s\right\|_{L^{\infty}\left(I ; L^{2}\right) \leqq} \leqq K_{2}\|f\|_{L^{r^{\prime}\left(I ; L^{q^{\prime}}\right)}}, \quad f \in L^{r^{\prime}}\left(I ; L^{q^{\prime}}\right) .
$$

Remark 2.1. (1) The constant $K_{2}$ in Lemma 2.2 does not depend on the interval $I$. (2.3) still holds with $I=\boldsymbol{R}$.

(2) In fact, a slightly stronger result holds than (2.3). That is,

$$
\int_{0}^{t} U(t-s) f(s) d s \in C\left(\bar{I} ; L^{2}\right)
$$

under the same assumptions as in Lemma 2.2, where $\bar{I}$ is the closure of $I$. This follows directly from the approximation of $f(t)$ by a sequence of smooth functions.

Lemma 2.2 is the version of Lemma 2.1 for the inhomogeneous linear Schrödinger equation. For the proof of Lemma 2.2, see [19, Lemmas 2.1 and 2.2].

We next state the local smoothing effect of the evolution operator for the free Schrödinger equation (see [4], [14] and [15]).

Lemma 2.3. Let $T>0$ and $\varphi \in C_{0}^{\infty}\left(\boldsymbol{R}^{N}\right)$.

(i) There exists a positive constant $K_{3}$ depending only on $N, T$ and $\varphi$ such that

$$
\left\|\varphi(1-\Delta)^{1 / 4} U(\cdot) u\right\|_{L^{2}\left(0, T ; L^{2}\right)} \leqq K_{3}\|v\|_{L^{2}}, \quad v \in L^{2} .
$$

(ii) There exists a positive constant $K_{4}$ depending only on $N, T$ and $\varphi$ such that

$$
\left\|\varphi(1-\Delta)^{1 / 4} \int_{0}^{t} U(t-s) f(s) d s\right\|_{L^{2}\left(0, T ; L^{2}\right) \leqq} K_{4}\|f\|_{L^{1}\left(0, T ; L^{2}\right)}, \quad f \in L^{1}\left(0, T ; L^{2}\right) .
$$

Proof. For the proof of Part (i), see [4, Theorem 2.1] and [14, (2) at page 701]. We briefly describe the proof of Part (ii). 
We use a duality argument to prove (ii). Let $\Omega$ be a bounded open set which includes the support of $\varphi$. Let $\Phi(t, x) \in C_{0}^{\infty}((0, T) \times \Omega)$. We denote $(1-\Delta)^{1 / 4}$ by $B$. Then, by using Schwarz's inequality and (2.5) we have

$$
\begin{aligned}
& \left|\int_{0}^{T}\left(B \int_{0}^{t} U(t-s) f(s) d s, \Phi(t)\right) d t\right| \\
& \quad=\left|\int_{0}^{T} \int_{0}^{t}(B U(t-s) f(s), \Phi(t)) d s d t\right| \\
& \quad \leqq \int_{0}^{T} \int_{0}^{t}\|B U(t-s) f(s)\|_{L^{2}(\Omega)}\|\Phi(t)\|_{L^{2}(\Omega)} d s d t \\
& \quad \leqq \int_{0}^{T} \int_{0}^{T}\|B U(t-s) f(s)\|_{L^{2}(\Omega)}\|\Phi(t)\|_{L^{2}(\Omega)} d t d s \\
& \quad \leqq \int_{0}^{T}\|B U(\cdot-s) f(s)\|_{L^{2}\left(0, T ; L^{2}(\Omega)\right)}\|\Phi\|_{L^{2}\left(0, T ; L^{2}(\Omega)\right)} d s \\
& \quad \leqq C \int_{0}^{T}\|U(-s) f(s)\|_{L^{2} d s\|\Phi\|_{L^{2}\left(0, T ; L^{2}(\Omega)\right)}} \\
& \quad \leqq C\|f\|_{L^{1}\left(0, T ; L^{2}\right)}\|\Phi\|_{L^{2}\left(0, T ; L^{2}(\Omega)\right)}
\end{aligned}
$$

This completes the proof of (ii), since $C_{0}^{\infty}((0, T) \times \Omega)$ is dense in $L^{2}(0, T$; $\left.L^{2}(\Omega)\right)$.

We finally state the lemma concerning the properties of the commutator $J_{k}(t), 1 \leqq k \leqq N$. This will be useful, when we consider the smoothing effect of (1.1)-(1.3) in the weighted Sobolev space.

Lemma 2.4. $J_{k}(t), 1 \leqq k \leqq N$, commute with $i \partial_{t}+\Delta$ and we have

$$
\begin{aligned}
& J_{k}(t)=M(t)\left(2 i t \partial_{k}\right) M(-t) \\
& J_{k}(t) U(t-s)=U(t-s) J_{k}(s)
\end{aligned}
$$

for $t, s \in \mathbb{R}$ and $1 \leqq k \leqq N$.

Lemma 2.4 follows from a direct calculation (see, e.g., [8] and [10]).

\section{§3. Proof of Theorem 1 .}

In this section we describe the proof of Theorem 1.1.

When we use the standard iteration scheme to solve (1.1)-(1.3), the loss of derivative occurs, as stated in $\S 1$. In fact, if $1 \leqq N \leqq 3$ and $E \in L^{\infty}\left(0, T ; H^{m}\right)$ for some $m \geqq 2$ and $T>0$, we solve (1.2) to have $n \in L^{\infty}\left(0, T ; H^{m-1}\right)$. However, we have only $E \in L^{\infty}\left(0, T ; H^{m-1}\right)$ by (1.1) when $n \in L^{\infty}\left(0, T ; H^{m-1}\right)$. 
Thus, we first consider the following system:

$$
\begin{aligned}
& i \partial_{t} F+\Delta F-n F-\partial_{t} n\left(E_{0}+\int_{0}^{t} F d s\right)=0, \\
& \partial_{t}^{2} n-\Delta n-\Delta|E|^{2}=0, \\
& (-\Delta+1) E=i F-(n-1)\left(E_{0}+\int_{0}^{t} F d s\right), \\
& F(0)=i\left(\Delta E_{0}-n_{0} E_{0}\right), \quad n(0)=n_{0}, \quad \partial_{t} n(0)=n_{1} .
\end{aligned}
$$

If we formally differentiate (1.1) in $t$ and put $F=\partial_{t} E$, we obtain (3.1). (1.1) is also rewritten as (3.3) in terms of $F$. The loss of derivative does not occur for (3.1)-(3.4). This technique was used to solve the fully nonlinear wave equation in [13].

Proposition 3.1. Assume that $1 \leqq N \leqq 3$. If $\left(E_{0}, n_{0}, n_{1}\right) \in H^{2} \oplus H^{1} \oplus L^{2}$, then for some $T>0$ there exist the solutions $(F(t), n(t))$ of (3.1)-(3.4) such that

$$
\begin{aligned}
& F \in\left[\bigcap_{j=0}^{1} C^{j}\left([0, T] ; H^{-2 j}\right)\right] \cap L^{8 / N}\left(0, T ; L^{4}\right), \\
& n \in \bigcap_{j=0}^{2} C^{j}\left([0, T] ; H^{1-j}\right), \\
& E \in C\left([0, T] ; H^{2}\right),
\end{aligned}
$$

where $T$ depends only on $N,\left\|E_{0}\right\|_{H^{2}},\left\|n_{0}\right\|_{H^{1}}$ and $\left\|n_{1}\right\|_{L^{2}}$. Furthermore, $E(t) \in$ $C^{1}\left([0, T] ; L^{2}\right), \frac{\partial}{\partial t} E(t)=F(t), E(0)=E_{0}$ and $(E(t), n(t))$ are the solutions of (1.1)(1.3) satisfying (1.6)-(1.8).

Proof. We put

$$
a=\max \left\{\left\|E_{0}\right\|_{L^{2}},\left\|E_{0}\right\|_{L^{4}},\left\|\Delta E_{0}-n_{0} E_{0}\right\|_{L^{2}},\left\|n_{0}\right\|_{H^{1}}+|| n_{1}\left\|_{L^{2}}+\right\| \omega\left|E_{0}\right|^{2} \|_{L^{2}}\right\} .
$$

We note by the Sobolev imbedding theorem that $a$ depends only on $N,\left\|E_{0}\right\|_{H^{2}}$, $\left\|n_{0}\right\|_{H^{1}}$ and $\left\|n_{1}\right\|_{L^{2}}$. (2.2) gives us

$$
\left\|U(\cdot) i\left(\Delta E_{0}-n_{0} E_{0}\right)\right\|_{L^{8 / \Delta}\left(\boldsymbol{R} ; L^{4}\right)} \leqq \delta a
$$

for some $\delta>0$. Since $U(t)$ is a unitary group in $L^{2}$, we have

$$
\left\|U(\cdot) i\left(\Delta E_{0}-n_{0} E_{0}\right)\right\|_{L^{\infty}\left(\boldsymbol{R} ; L^{2}\right)} \leqq a .
$$

Let $T$ be a small positive constant to be determined later. We put $I=(0, T)$. We define the Banach space $X$ and its norm $\||\cdot|\|$ as follows:

$$
X=\left[L^{\infty}\left(I ; L^{2}\right) \cap L^{8 / N}\left(I ; L^{4}\right)\right] \oplus\left[L^{\infty}\left(I ; H^{1}\right) \cap W^{1, \infty}\left(I ; L^{2}\right)\right]
$$


and

$$
\|(F, n)\|\|=\| F\left\|_{L^{\infty}\left(I ; L^{2}\right)}+\right\| F\left\|_{L^{8 / N}\left(I ; L^{4}\right)}+\right\| n\left\|_{L^{\infty}\left(I ; H^{1}\right)}+\right\| \frac{d}{d t} n \|_{L^{\infty}\left(I ; L^{2}\right)} .
$$

We put

$$
\begin{array}{r}
Y=\left\{(F, n) \in X ;\|F\|_{L^{\infty}\left(I ; L^{2}\right) \leqq} \leqq,\|F\|_{L^{8 / L}\left(I ; L^{4}\right)} \leqq 2 \delta a,\right. \\
\left.\|n\|_{L^{\infty}\left(I ; H^{1}\right)} \leqq 2 a,\left\|\frac{d}{d t} n\right\|_{L^{\infty}\left(I ; L^{2}\right)} \leqq 2 a\right\} .
\end{array}
$$

We note that $Y$ is a closed subset in $X$. For $(F, n) \in Y$ we define the nonlinear mapping $N[F, n](t)$ as follows:

$$
\begin{gathered}
N[F, n](t)=\left(N_{1}[F, n](t), N_{2}[F, n](t)\right), \\
N_{1}[F, n](t)=U(t) i\left(\Delta E_{0}-n_{0} E_{0}\right) \\
+\int_{0}^{t} U(t-s)\left\{n(s) F(s)+\partial_{s} n(s)\left(E_{0}+\int_{0}^{s} F d \tau\right)\right\} d s, \\
N_{2}[F, n](t)=\cos \omega t n_{0}+\omega^{-1} \sin \omega t n_{1} \\
+\int_{0}^{t} \omega^{-1} \sin \omega(t-s) \Delta|E(s)|^{2} d s,
\end{gathered}
$$

where

$$
E(t)=(-\Delta+1)^{-1}\left\{i F-(n-1)\left(E_{0}+\int_{0}^{t} F(s) d s\right)\right\}
$$

Since the fixed points $(F, n)$ of $N[F, n]$ are the solutions of (3.1)-(3.4), we show that for sufficiently small $T>0 N[F, n]$ is a contraction mapping from $Y$ into $Y$.

We first show that for sufficiently small $T>0 N[F, n]$ is a mapping from $Y$ into $Y$. Let $(F, n) \in Y$. We take the $L^{4}$ norm of (3.10) and use Lemma 2.1(i) with $p=4$ and Hölder's inequality with $3 / 4=1 / 2+1 / 4$ to obtain

$$
\begin{aligned}
\left\|N_{1}[F, n](t)\right\|_{L^{4}} \leqq\left\|U(t) i\left(\Delta E_{0}-n_{0} E_{0}\right)\right\|_{L^{4}} \\
+C \int_{0}^{t}|t-s|^{-N / 4}\left\{\|n\|_{L^{2}}\|F\|_{L^{4}}\right. \\
\left.+\left\|\frac{d}{d t} n\right\|_{L^{2}}\left(\left\|E_{0}\right\|_{L^{4}}+\int_{0}^{s}\|F\|_{L^{4}} d \tau\right)\right\} d s .
\end{aligned}
$$

We take the $L^{8 / N}(I)$ norm of (3.13) and use the Hardy-Littlewood-Sobolev inequality and (3.8) to obtain 


$$
\begin{aligned}
&\left\|N_{1}[F, n]\right\|_{L^{8 / N}\left(I ; L^{4}\right) \leqq} \leqq \\
&+C T^{1-N / 4}\|n\|_{L^{\infty}\left(I ; L^{2}\right)}\|F\|_{L^{8 / \Delta}\left(I ; L^{4}\right)} \\
&+C T^{1-N / 8}\left\|\frac{d}{d t} n\right\|_{L^{\infty}\left(I ; L^{2}\right)}\left\|E_{0}\right\|_{L^{4}} \\
&+C T^{2-N / 8}\left\|\frac{d}{d t} n\right\|_{L^{\infty}\left(I ; L^{2}\right)}\|F\|_{L^{8 / N}\left(I ; L^{4}\right)} \\
& \leqq \delta a+C\left(T^{1-4 / N} a+T^{1-N / 8} \delta^{-1} a+T^{2-N / 4} a\right) \delta a .
\end{aligned}
$$

If we choose $T>0$ so small that

$$
C\left(T^{1-N / 4} a+T^{1-N / 8} \delta^{-1} a+T^{2-N / 4} a\right) \leqq 1,
$$

then (3.14) gives us

$$
\left\|N_{1}[F, n]\right\|_{L^{8 / \Delta}\left(I ; L^{4}\right)} \leqq 2 \delta a .
$$

We next take the $L^{\infty}\left(I ; L^{2}\right)$ norm of (3.10) and use Lemma 2.2(i) with $q^{\prime}=4 / 3$ and $r^{\prime}=8 /(8-N)$ and (3.9) to obtain

$$
\begin{aligned}
& \left\|N_{1}[F, n]\right\|_{L^{\infty}\left(I ; L^{2}\right)} \leqq a \\
& +C T^{1-N / 4}\|n\|_{L^{\infty}\left(I ; L^{2}\right)}\|F\|_{L^{8 / N}\left(I ; L^{4}\right)} \\
& +C T^{1-N / 8}\left\|\frac{d}{d t} n\right\|_{L^{\infty}\left(I ; L^{2}\right)}\left\|E_{0}\right\|_{L^{4}} \\
& +C T^{2-N / 4}\left\|\frac{d}{d t} n\right\|_{L^{\infty}\left(I ; L^{2}\right)}\|F\|_{L^{8 / D}\left(I ; L^{4}\right)} \\
& \leqq a+C\left(T^{1-N / 4} \delta a+T^{1-N / 8} a+T^{2-N / 4} \delta a\right) a \text {. }
\end{aligned}
$$

If we choose $T>0$ so small that

$$
C\left(T^{1-N / 4} \delta a+T^{1-N / 8} a+T^{2-N / 4} \delta a\right) \leqq 1,
$$

then (3.16) gives us

$$
\left\|N_{1}[F, n]\right\|_{L^{\infty}\left(I ; L^{2}\right) \leqq 2 a} .
$$

By (3.12) we evaluate $E$ to obtain

$$
\begin{aligned}
& \|E\|_{L^{\infty}\left(I ; H^{2}\right) \leqq C} \leqq F \|_{L^{\infty}\left(I ; L^{2}\right)} \\
& \quad+\|n\|_{L^{\infty}\left(I ; L^{4}\right)}\left(\left\|E_{0}\right\|_{L^{4}}+\int_{0}^{T}\|F\|_{L^{4}} d s\right) \\
& \left.\quad+\left\|E_{0}\right\|_{L^{2}}+\int_{0}^{T}\|F\|_{L^{2}} d s\right] \\
& \leqq C\left(a+a^{2}+T^{1-N / 8} \delta a^{2}+T^{1-N / 8} \delta a\right), \quad(F, n) \in Y .
\end{aligned}
$$


Here we have used the Sobolev imbedding theorem at the second inequality.

Accordingly, we have by (3.11) and (3.18)

$$
\begin{aligned}
& \left\|N_{2}[F, n](t)\right\|_{H^{1}} \leqq(1+t) a \\
& \quad+\int_{0}^{t}(1+s)\left\|\Delta|E(s)|^{2}\right\|_{L^{2}} d s \\
& \leqq \\
& \quad(1+T) a \\
& \quad+C T(1+T)\left(a+a^{2}+T^{1-N / 8} \delta a^{2}+T^{1-N / 8} \delta a\right)^{2} \\
& =a+\left[T+C T(1+T)\left(1+a+T^{1-N / 8} \delta a+T^{1-N / 8} \delta\right)^{2} a\right] a, t \in \mathbb{I} .
\end{aligned}
$$

If we choose $T>0$ so small that

$$
T+C T(1+T)\left(1+a+T^{1-N / 8} \delta a+T^{1-N / 8} \delta\right) a \leqq \mathbb{1},
$$

then (3.19) yields

$$
\left\|N_{2}[F, n]\right\|_{L^{\infty}\left(I ; H^{1}\right)} \leqq 2 a .
$$

On the other hand, we have

$$
\begin{aligned}
& \frac{d}{d t} N_{2}[F, n](t)=-\omega \sin \omega t n_{0} \\
& \quad+\cos \omega t n_{1}+\omega^{-1} \sin \omega t \Delta\left|E_{0}\right|^{2} \\
& +\int_{0}^{t} \cos \omega(t-s) \Delta|E(s)|^{2} d s
\end{aligned}
$$

Therefore, we take the $L^{2}$ norm of (3.21) and use the Sobolev imbedding theorem and (3.18) to obtain

$$
\begin{aligned}
& \left\|\frac{d}{d t} N_{2}[F, n](t)\right\|_{L^{2}} \\
& \quad \leqq a+C \int_{0}^{t}\left\|\Delta|E(s)|^{2}\right\|_{L^{2}} d s \\
& \quad \leqq a+C T\|E\|_{L^{\infty}\left(I ; H^{2}\right)}^{2} \\
& \quad \leqq a+C T\left(1+a+T^{1-N / 8} \delta a+T^{1-N 8} \delta\right)^{2} a^{2}, \quad t \in I .
\end{aligned}
$$

If we choose $T>0$ so small that

$$
C T\left(1+a+T^{1-N / 8} \delta a+T^{1-N / 8} \delta\right)^{2} a \leqq 1,
$$

then (3.22) yields

$$
\left\|\frac{d}{d t} N_{2}[F, n](t)\right\|_{L^{2}} \leqq 2 a
$$


Therefore, (3.15), (3.17), (3.20) and (3.23) show that for sufficiently small $T>0 N[F, n]$ is a mapping from $Y$ into $Y$. In the same way as above we obtain

$$
\begin{array}{r}
\left|\left\|N[F, n]-N\left[F^{\prime}, n^{\prime}\right] \mid\right\| \leqq \frac{1}{2}\left\|(F, n)-\left(F^{\prime}, n^{\prime}\right)\right\| \|,\right. \\
(F, n), \quad\left(F^{\prime}, n^{\prime}\right) \in Y
\end{array}
$$

for sufficiently small $T>0$, which implies that $N[F, n]$ is a contraction mapping from $Y$ into $Y$. Accordingly, there exist the unique fixed points $(F, n)$ of $N[F, n]$ and $E(t)$ is determined by $(F, n)$ in terms of (3.12). These $(F, n, E)$ satisfy (3.1)-(3.4) in the integral form and

$$
\begin{aligned}
& F \in L^{\infty}\left(I ; L^{2}\right) \cap L^{8 / N}\left(0, T ; L^{4}\right), \\
& n \in \bigcap_{j=0}^{1} W^{j, \infty}\left(I ; H^{1-j}\right), \\
& E \in L^{\infty}\left(I ; H^{2}\right) .
\end{aligned}
$$

(3.25)-(3.27) and the standard argument show that $(F, n, E)$ are the solutions of (3.1)-(3.4) satisfying (3.5)-(3.7) (see, e.g., Remark 2.1(2)).

We next prove that $E \in C^{1}\left([0, T] ; L^{2}\right)$ and $\frac{d}{d t} E(t)=F(t)$. We differentiate (3.3) in $t$ to obtain

$$
\begin{aligned}
(-\Delta+1) \frac{d}{d t} E= & i \frac{d}{d t} F-(n-1) F \\
& -\frac{d}{d t} n\left(E_{0}+\int_{0}^{t} F d s\right) \text { in } H^{-2} .
\end{aligned}
$$

On the other hand, (3.1) gives us

$$
\begin{aligned}
(-\Delta+1) F= & i \frac{d}{d t} F-(n-1) F \\
& -\frac{d}{d t} n\left(E_{0}+\int_{0}^{t} F d s\right) \text { in } H^{-2} .
\end{aligned}
$$

Therefore, $\frac{d}{d t} E(t)=F(t)$ in $H^{-2}$. Furthermore, by (3.28) we have

$$
\begin{aligned}
& \frac{d}{d t} E(t)=(-\Delta+1)^{-1}\left[i \frac{d}{d t} F-(n-1) F\right. \\
&\left.+\frac{d}{d t} n\left(E_{0}+\int_{0}^{t} F d s\right)\right] .
\end{aligned}
$$


The right hand side of $(3.30)$ is in $C\left([0, T] ; L^{2}\right)$. Accordingly, $E(t) \in$ $C^{1}\left([0, T] ; L^{2}\right) . \quad$ By (3.3) and (3.4) we have

$$
\begin{aligned}
(-\Delta+1) E(0) & =i F_{0}-\left(n_{0}-1\right) E_{0} \\
& =i \cdot i\left(\Delta E_{0}-n_{0} E_{0}\right)-\left(n_{0}-1\right) E_{0} \\
& =(-\Delta+1) E_{0},
\end{aligned}
$$

which shows $E(0)=E_{0}$.

It remains only to show that $E \in L^{8 / N}\left(0, T ; W^{2,4}\right)$, which follows directly from (1.1), (3.5) and the regularity theorem of the elliptic equation.

Therefore, $(E(t), n(t))$ are the unique solutions of (1.1)-(1.3) satisfying (1.6)-(1.8).

In the same way as in the proof of Proposition 3.1 we have the following proposition.

Proposition 3.2. Assume that $1 \leqq N \leqq 3$.

(1) Let $m$ be an even integer with $m \geqq 4$. If $\left(E_{0}, n_{0}, n_{1}\right) \in H^{m} \oplus H^{m-1} \oplus H^{m-2}$, then for some $\tilde{\mathbb{T}}>0$ there exist the unique solutions $(E(t), n(t))$ of $(1.1)-(1.3)$ satisfying (1.9)-(1.12) with $T$ replaced by $\tilde{T}$, where $\tilde{T}$ depends only on $N,\left\|E_{0}\right\|_{H^{m}},\left\|n_{0}\right\|_{H^{m-1}}$ and $\left\|n_{1}\right\|_{H^{m-2}}$ 。

(2) Let $m$ be an odd integer with $m \geqq 3$. If $\left(E_{0}, n_{0}, n_{1}\right) \in H^{m} \oplus H^{m-1} \oplus H^{m-2}$, then for some $\tilde{T}>0$ there exist the unique solutions $(E(t), n(t))$ of $(1.1)-(1.3)$ satisfying (1.13)-(1.16) with $T$ replaced by $\widetilde{T}$. where $\widetilde{T}$ depends only on $N,\left\|E_{0}\right\|_{H^{m}},\left\|n_{0}\right\|_{H^{m-1}}$ and $\left\|n_{1}\right\|_{H^{m-2}}$.

Remark 3.1. We note that in Proposition 3.2 $\widetilde{T}$ depends on the higher order Sobolev norms of $\left(E_{0}, n_{0}, n_{1}\right)$ than the norms of $H^{2} \oplus H^{1} \oplus L^{2}$ and that $\tilde{T}$ is less than $T$ given by Proposition 3.1.

We omit the proof of Proposition 3.2, since it is similar to the proof of Proposition 3.1 .

We can complete the proof of Theorem 3.1(2)-(3), if we prove the following proposition.

Proposition 3.3. Assume that $1 \leqq N \leqq 3$. Let $m$ be an integer with $m \geqq 2$ and let $\left(E_{0}, n_{0}, n_{1}\right) \in H^{m+1} \oplus H^{m} \oplus H^{m-1}$. If Theorem 1.1 holds for $m$, then Theorem 1.1 also holds for $m+1$.

Proof. Let $(E(t), n(t))$ be the solutions of (1.1)-(1.3) satisfying Theorem 1.1 for $m$. Since $\left(E_{0}, n_{0}, n_{1}\right) \in H^{m+1} \oplus H^{m} \oplus H^{m-1}$, by Proposition 3.2 and the 
uniqueness of solutions we conclude that $(E(t), n(t))$ satisfy Theorem 1.1 for $m+1$ with $T$ replaced by $\widetilde{T}$.

We prove Proposition 3.3 only in the case where $m$ is even, since the proof for odd $m$ is the same. Let $\widetilde{T}_{m a x}$ be the maximal existence time of the solutions $(E(t), n(t))$ in $H^{m+1} \oplus H^{m}$. That is, for any $T^{\prime}$ with $0<T^{\prime}<\widetilde{T}_{\max }(E(t), n(t))$ satisfy (1.13)-(1.16) with $T$ and $m$ replaced by $T^{\prime}$ and $m+1$. If $\widetilde{T}_{\max }>T$, the proof is completed. We suppose that $\widetilde{T}_{m a x} \leqq T$ and derive a contradiction. We divide the proof into three cases.

Case 1 . Let $m$ be an even integer with $m \geqq 6$. Since $(E(t), n(t))$ satisfy (1.13)-(1.16) with $T$ and $m$ replaced by $T^{\prime}$ and $m+1$ for any $T^{\prime}$ with $0<T^{\prime}<\widetilde{T}_{m a x}$, we differentiate (1.1) $m / 2-1$ times in $t$ and once in $x_{k}, 1 \leqq k \leqq N$ to obtain

$$
\begin{aligned}
i \partial_{t}\left(\partial_{k} \partial_{t}^{m / 2-1} E\right)+\Delta\left(\partial_{k} \partial_{t}^{m / 2-1} E\right) & \\
= & \sum_{j=0}^{m / 2-1}\left(\begin{array}{c}
m / 2-1 \\
j
\end{array}\right)\left[\left(\partial_{k} \partial_{t}^{m / 2-1-j} n\right)\left(\partial_{t}^{j} E\right)+\left(\partial_{t}^{m / 2-1-j} n\right)\left(\partial_{k} \partial_{t}^{j} E\right)\right] \\
& \quad \text { in } L^{2}, \quad 1 \leqq k \leqq N
\end{aligned}
$$

for $0 \leqq t<\tilde{T}_{\text {max }}$. By the assumption that Theorem 1.1 holds for $m$, we have

$$
\begin{aligned}
& \partial_{t}^{m / 2-1-j} n \in L^{\infty}\left(0, T ; H^{4}\right), \quad 0 \leqq j \leqq m / 2-2, \\
& n \in L^{\infty}\left(0, T ; H^{5}\right), \\
& \partial_{t}^{j} E \in L^{\infty}\left(0, T ; H^{4}\right), \quad 0 \leqq j \leqq m / 2-2, \\
& \partial_{t}^{m / 2-1} E \in L^{\infty}\left(0, T ; L^{2}\right) .
\end{aligned}
$$

Therefore, by (3.32)-(3.36) and the Sobolev imbedding theorem we obtain

$$
\begin{aligned}
& \left\|\partial_{k} \partial_{t}^{m / 2-1} E(t)\right\|_{H^{2}} \leqq\left\|\partial_{k} \partial_{t}^{m / 2-1} E(0)\right\|_{H^{2}} \\
& +C \int_{0}^{t} \sum_{j=0}^{m / 2-2}\left[\left\|\left(\partial_{k} \partial_{t}^{m / 2-1-j} n\right)\left(\partial_{t}^{j} E\right)\right\|_{H^{2}}\right. \\
& \left.+\left\|\left(\partial_{t}^{m / 2-1-j} n\right)\left(\partial_{k} \partial_{t}^{j} E\right)\right\|_{H^{2}}\right] d \tau \\
& +C \int_{0}^{t}\left\|\left(\partial_{k} n\right)\left(\partial_{t}^{m / 2-1} E\right)\right\|_{H^{2}} d \tau \\
& +C \int_{0}^{t}\left\|n \partial_{k} \partial_{t}^{m / 2-1} E\right\|_{H^{2}} d \tau \\
& \leqq\left\|\partial_{k} \partial_{t}^{m / 2-1} E(0)\right\|_{H^{2}} \\
& +C \int_{0}^{T} \sum_{j=0}^{m / 2-1}\left[\left\|\partial_{t}^{m / 2-1-j} n\right\|_{H^{4}}\left\|\partial_{t}^{j} E\right\|_{H^{3}}\right. \\
& \left.+\left\|\partial_{t}^{m / 2-1-j} n\right\|_{H^{3}}\left\|\partial_{t}^{j} E\right\|_{H^{4}}\right] d \tau
\end{aligned}
$$




$$
\begin{array}{r}
+C \int_{0}^{T}\|n\|_{H^{5}}\left\|\partial_{t}^{m / 2-1} E\right\|_{H^{2}} d \tau \\
+C \int_{0}^{t}\|n\|_{H^{4}}\left\|\partial_{t}^{m / 2-1} E\right\|_{H^{2}} d \tau \\
\leqq C+C \int_{0}^{t}\left\|\partial_{k} \partial_{t}^{m / 2-1} E\right\|_{H^{2}} d \tau
\end{array}
$$

for $t \in\left[0, \widetilde{T}_{m a x}\right)$ and $1 \leqq k \leqq N$. (3.37) and Gronwall's inequality yield

$$
\partial_{k} \partial_{t}^{m / 2-1} E \in L^{\infty}\left(0, \widetilde{\mathbb{T}}_{\max } ; H^{2}\right), \quad 1 \leqq k \leqq N .
$$

By differentiating (1.1) $m / 2-2$ times in $t$, we have

$$
\begin{aligned}
i \partial_{t}\left(\partial_{t}^{m / 2-2} E\right)+\Delta\left(\partial_{t}^{m / 2-2} E\right) & \\
= & \sum_{j=0}^{m / 2-2}\left(\begin{array}{c}
m / 2-2 \\
j
\end{array}\right)\left(\partial_{t}^{m / 2-2} n\right)\left(\partial_{t}^{j} E\right) .
\end{aligned}
$$

Since Theorem 1.1 holds for $m$, we easily see by the Sobolev imbedding theorem that the right hand side of (3.39) belongs to $L^{\infty}\left(0, \widetilde{T}_{\text {max }} ; H^{3}\right)$. Therefore, by (3.39), (3.38) and the regularity theorem of the elliptic equation we obtain

$$
\partial_{8}^{m / 2-2} E \in L^{\infty}\left(0, \widetilde{T}_{\text {max }} ; H^{5}\right)
$$

Repeating this procedure, we conclude that

$$
E \in L^{\infty}\left(0, \widetilde{T}_{\max } ; H^{m+1}\right)
$$

(3.41) and (3.2) imply that

$$
\begin{aligned}
& n \in L^{\infty}\left(0, \widetilde{T}_{\text {max }} ; H^{m}\right), \\
& \partial_{t} n \in L^{\infty}\left(0, \widetilde{T}_{\text {max }} ; H^{m-1}\right) .
\end{aligned}
$$

(3.41)-(3.43) assure that by Proposition 3.2 we can extend the solutions $(E(t)$ $n(t))$ as the solutions in $H^{m+1} \oplus H^{m}$ beyond $t=\widetilde{T}_{m a x}$. This contradicts the definition of $\widetilde{\mathbb{T}}_{\text {max }}$.

Case 2. Let $m=4$. Since $(E(t), n(t))$ satisfy $(1.13)-(1.16)$ with $T$ and $m$ replaced by $T^{\prime}$ and $m+1$ for any $T^{\prime}$ with $0<T^{\prime}<\widetilde{T}_{\max }$, we have by (1.1)

$$
E \in C^{3}\left(\left[0, \widetilde{T}_{m a x}\right) ; H^{-1}\right) .
$$

We differentiate (1.1) twice in $t$ and once in $x_{k}, 1 \leqq k \leqq N$ to obtain 


$$
\begin{aligned}
& i \partial_{t}\left(\partial_{k} \partial_{t}^{2} E\right)+\Delta\left(\partial_{k} \partial_{t}^{2} E\right) \\
& =\sum_{j=0}^{2}\left(\begin{array}{l}
2 \\
j
\end{array}\right)\left[\left(\partial_{k} \partial_{t}^{2-j} n\right)\left(\partial_{t}^{j} E\right)+\left(\partial_{t}^{2-j} n\right)\left(\partial_{k} \partial_{t}^{j} n\right)\right] \\
& \quad \text { in } H^{-2}, \quad 0 \leqq t<\widetilde{T}_{\max }, \quad 1 \leqq k \leqq N .
\end{aligned}
$$

We rewrite (3.45) as the integral form:

$$
\begin{aligned}
\partial_{k} \partial_{t}^{2} E(t)= & U(t) \partial_{k} \partial_{t}^{2} E(0) \\
& -i \int_{0}^{t} U(t-s)\left[\sum_{j=0}^{2}\left(\begin{array}{l}
2 \\
j
\end{array}\right)\left\{\left(\partial_{k} \partial_{s}^{2-j} n\right)\left(\partial_{s}^{j} E\right)+\left(\partial_{s}^{2-j} n\right)\left(\partial_{k} \partial_{s}^{j} E\right)\right\}\right] d s, \\
& 0 \leqq t<\tilde{T}_{\max }, \quad 1 \leqq k \leqq N
\end{aligned}
$$

We take the $L^{4}$ norm of (3.46) and use Lemma 2.1(i) to obtain

$$
\begin{aligned}
\left\|\partial_{k} \partial_{t}^{2} E(t)\right\|_{L^{4}} \leqq & \left\|U(t) \partial_{k} \partial_{t}^{2} E(0)\right\|_{L^{4}} \\
& +C \sum_{j=0}^{2} \int_{0}^{t}|t-s|^{-N / 4}\left[\left\|\left(\partial_{k} \partial_{s}^{2-j} n\right)\left(\partial_{s}^{j} E\right)\right\|_{L^{4 / 3}}\right. \\
& \left.\quad+\left\|\left(\partial_{s}^{2-j} n\right)\left(\partial_{k} \partial_{s}^{i} E\right)\right\|_{L^{4} / 3}\right] d s \\
\leqq & \left\|U(t) \partial_{k} \partial_{t}^{2} E(0)\right\|_{L^{4}} \\
& +C \sum_{j=0}^{1} \int_{0}^{t}|t-s|^{-N / 4}\left[\left\|\partial_{k} \partial_{s}^{2-j} n\right\|_{L^{2}}\left\|\partial_{s}^{j} E\right\|_{L^{4}}\right. \\
& \left.+\left\|\partial_{s}^{2-j} n\right\|_{L^{2}}\left\|\partial_{k} \partial_{s}^{j} E\right\|_{L^{4}}\right] d s \\
+ & C \int_{0}^{t}|t-s|^{-N / 4}\left\|\partial_{k} n\right\|_{L^{4}}\left\|\partial_{s}^{2} E\right\|_{L^{2}} d s \\
+ & C \int_{0}^{t}|t-s|^{-N / 4}\|n\|_{L^{4}}\left\|\partial_{k} \partial_{s}^{j} E\right\|_{L^{4}} d s \\
& 0 \leqq t<\widetilde{T}_{m a x}, \quad 1 \leqq k \leqq N .
\end{aligned}
$$

By the definition of $\widetilde{T}_{\text {max }}$ we note that $\partial_{k} \partial_{t}^{2} E \in L^{8 / N}\left(0, T^{\prime} ; L^{4}\right)$ for any $T^{\prime}$ with $0<T^{\prime}<\widetilde{T}_{m a x}$. By the assumption that Theorem 1.1 holds for $m=4$, we have

$$
\begin{array}{ll}
\partial_{t}^{2-j} n \in L^{\infty}\left(0, T ; H^{3-j}\right), & 0 \leqq j \leqq 2, \\
\partial_{t}^{j} E \in L^{\infty}\left(0, T ; H^{4-2 j}\right), & 0 \leqq j \leqq 2 .
\end{array}
$$

Therefore, by (3.47)-(3.49) and the Sobolev imbedding theorem we obtain

$$
\begin{gathered}
\left\|\partial_{k} \partial_{t}^{2} E(t)\right\|_{L^{4} \leqq}\left\|U(t) \partial_{k} \partial_{t}^{2} E(0)\right\|_{L^{4}} \\
+C+C \int_{0}^{t}|t-s|^{-N / 4}\left\|\partial_{k} \partial_{s}^{j} E(s)\right\|_{L^{4}} d s, \\
0 \leqq t<\tilde{T}_{m a x}, \quad 1 \leqq k \leqq N .
\end{gathered}
$$

By Lemma 2.1(ii) with $q=4$ and $r=8 / N$ we have 


$$
\left\|U(\cdot) \partial_{k} \partial_{b}^{2} E(0)\right\|_{L^{4} \in L^{8 / N}(\boldsymbol{R}) .}
$$

Accordingly, (3.50), (3.51) and the theory of the Volterra type integral equation show that

$$
\partial_{k} \partial_{t}^{2} E \in L^{8 / N}\left(0, \widetilde{T}_{m a x} ; L^{4}\right), \quad 1 \leqq k \leqq N .
$$

We next take the $L^{\infty}\left(0, \tilde{T}_{\text {max }} ; L^{2}\right)$ norm of (3.46) and use Lemma 2.2 with $q^{\prime}=4 / 3$ and $r^{\prime}=8 /(8-N)$ to obtain by (3.48) and (3.49)

$$
\left\|\partial_{k} \partial_{8}^{2} E\right\|_{L^{\infty}\left(0, \widetilde{T}_{\max } ; L^{2}\right)} \leqq C+C\left\|\partial_{k} \partial_{t}^{2} E\right\|_{L^{8 / N}\left(0, \tilde{T}_{\max } ; L^{4}\right)}, \quad 1 \leqq k \leqq N
$$

in the same way as (3.47). (3.53) and (3.52) show that

$$
\partial_{k} \partial_{s}^{2} E \in L^{\infty}\left(0, \widetilde{T}_{m a x} ; L^{2}\right), \quad 1 \leqq k \leqq N .
$$

We differentiate (1.1) in $t$ to obtain

$$
i \partial_{t}^{2} E+\Delta\left(\partial_{t} E\right)=\left(\partial_{t} n\right) E+n \partial_{t} E \text { in } L^{2}, \quad 0 \leqq t<\widetilde{T}_{\max } .
$$

(3.48), (3.49) and the Sobolev imbedding theorem imply that the right hand side of (3.55) belongs to $L^{\infty}\left(0, T ; H^{1}\right)$. Accordingly, (3.54), (3.55) and the regularity theorem of the elliptic equation give us

$$
\partial_{t} E \in L^{\infty}\left(0, \widetilde{T}_{m a x} ; H^{3}\right) .
$$

Repeating this procedure, we have

$$
E \in L^{\infty}\left(0, \widetilde{T}_{m a x} ; H^{5}\right) .
$$

(3.57) and (1.2) imply that

$$
\begin{aligned}
& n \in L^{\infty}\left(0, \widetilde{T}_{m a x} ; H^{4}\right), \\
& \partial_{t} n \in L^{\infty}\left(0, \widetilde{T}_{m a x} ; H^{3}\right) .
\end{aligned}
$$

(3.57)-(3.59) assure that by Proposition 3.2 we can extend the solutions $(E(t), n(t))$ as the solutions in $H^{5} \oplus H^{4}$ beyond $t=\tilde{T}_{\text {max }}$. This contradicts the definition of $\tilde{T}_{\max }$.

Case 3. Let $m=2$. Since $(E(t), n(t))$ satisfy (1.13)-(1.15) with $T$ and $m$ replaced by $T^{\prime}$ and $m+1$ for any $T^{\prime}$ with $0<T^{\prime}<\widetilde{T}_{\max }$, we have by (1.1)

$$
E \in C^{2}\left(\left[0, \widetilde{T}_{m a x}\right) ; H^{-1}\right) .
$$

We differentiate (1.1) in $t$ and $x_{k}, 1 \leqq k \leqq N$ to obtain 


$$
\begin{aligned}
i \partial_{t}\left(\partial_{k} \partial_{t} E\right)+\Delta\left(\partial_{k} \partial_{t} E\right)= & \left(\partial_{k} \partial_{t} n\right) E+\left(\partial_{t} n\right)\left(\partial_{k} E\right) \\
& +\left(\partial_{k} n\right)\left(\partial_{t} E\right)+n\left(\partial_{k} \partial_{t} E\right) \text { in } H^{-2}
\end{aligned}
$$

for $0 \leqq t<\widetilde{T}_{\max }$ and $1 \leqq k \leqq N$. We rewrite (3.61) as the integral form:

$$
\begin{aligned}
\partial_{k} \partial_{t} E(t)= & U(t) \partial_{k} \partial_{t} E(0) \\
& -i \int_{0}^{t} U(t-s)\left[\left(\partial_{k} \partial_{s} n\right) E+\left(\partial_{s} n\right)\left(\partial_{k} E\right)\right. \\
& \left.+\left(\partial_{k} n\right)\left(\partial_{s} E\right)+n\left(\partial_{k} \partial_{s} E\right)\right] d s \\
& 0 \leqq t<\widetilde{T}_{\text {max }}, \quad 1 \leqq k \leqq N .
\end{aligned}
$$

We take the $L^{4}$ norm of (3.62) and use Lemma 2.1(i) to obtain

$$
\begin{aligned}
& \left\|\partial_{k} \partial_{t} E(t)\right\|_{L^{4}} \leqq\left\|U(t) \partial_{k} \partial_{t} E(0)\right\|_{L^{4}} \\
& +C \int_{0}^{t}|t-s|^{-N / 4}\left[\left\|\left(\partial_{k} \partial_{s} n\right) E\right\|_{L^{4 / 3}}\right.
\end{aligned}
$$

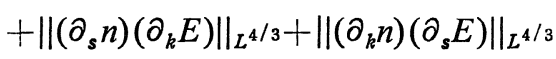

$$
\begin{aligned}
& +\left\|n\left(\partial_{k} \partial_{s} E\right)\right\|_{\left.L^{4 / 3}\right] d s} \\
& \leqq\left\|U(t) \partial_{k} \partial_{t} E(0)\right\|_{L^{4}} \\
& +C \int_{0}^{t}|t-s|^{-N / 4}\left[\left\|\partial_{k} \partial_{s} n\right\|_{L^{2}}\|E\|_{L^{4}}\right. \\
& +\left\|\partial_{s} n\right\|_{L^{2}}\left\|\partial_{k} E\right\|_{L^{4}}+\left\|\partial_{k} n\right\|_{L^{2}}\left\|\partial_{s} E\right\|_{L^{4}} \\
& \left.+\|n\|_{L^{2}}\left\|\partial_{k} \partial_{s} E\right\|_{L^{4}}\right] d s \text {, } \\
& 0 \leqq t<\widetilde{T}_{m a x}, \quad 1 \leqq k \leqq N .
\end{aligned}
$$

By the definition of $\widetilde{T}_{m a x}$ we note that $\partial_{k} \partial_{t} E \in L^{8 / N}\left(0, T^{\prime} ; L^{4}\right)$ for any $T^{\prime}$ with $0<T^{\prime}<\widetilde{T}_{m a x}$. By the assumption that Theorem 1.1 holds for $m$, we have

$$
\begin{aligned}
& \partial_{t}^{j} n \in L^{\infty}\left(0, T ; H^{1-j}\right), \quad j=0,1, \\
& \partial_{i}^{j} E \in L^{\infty}\left(0, T ; H^{2-2 j}\right), \quad j=0,1, \\
& \partial_{t}^{j} E \in L^{8 / N}\left(0, T ; W^{2-2 j, 4}\right), \quad j=0,1 .
\end{aligned}
$$

(3.63)-(3.66) and the Sobolev imbedding theorem give us

$$
\begin{aligned}
\left\|\partial_{k} \partial_{t} E(t)\right\|_{L^{4}} \leqq & \left\|U(t) \partial_{k} \partial_{t} E(0)\right\|_{L^{4}} \\
& +C+C \int_{0}^{t}|t-s|^{-N / 4}|| \partial_{s} E \|_{L^{4}} d s \\
& +C \int_{0}^{t}|t-s|^{-N / 4}\left(\left\|\partial_{k} \partial_{s} n\right\|_{L^{2}}+\left\|\partial_{k} \partial_{s} E\right\|_{L^{4}}\right) d s, \\
& 0 \leqq t<\widetilde{T}_{m a x}, \quad 1 \leqq k \leqq N .
\end{aligned}
$$


On the other hand, by (1.2) we have

$$
\begin{aligned}
\partial_{s}^{2} n-\Delta n & =\Delta|E|^{2} \\
& =\sum_{j=1}^{N} 2\left(\left|\nabla E_{j}\right|^{2}+\operatorname{Re} \Delta E_{j} \bar{E}_{j}\right) .
\end{aligned}
$$

Since we have by (1.1)

$$
\Delta E_{j}=-i \partial_{t} E_{j}+n E_{j}, \quad 1 \leqq j \leqq N,
$$

we obtain by (3.68)

$$
\partial_{t}^{2} n-\Delta n=2 \sum_{j=1}^{N}\left|\nabla E_{j}\right|^{2}+2 \sum_{j=1}^{N} \operatorname{Im} \partial_{t} E_{j} \bar{E}_{j}+n|E|^{2} .
$$

(3.69) yields

$$
\begin{aligned}
\left\|\partial_{k} \nabla n(t)\right\|_{L^{2}}+ & \left\|\partial_{k} \partial_{t} n(t)\right\|_{L^{2}} \\
\leqq & C\left(\left\|\partial_{k} \nabla n_{0}\right\|_{L^{2}}+\left\|\partial_{k} n_{1}\right\|_{L^{2}}\right) \\
& +C \int_{0}^{t} \sum_{j=1}^{N}\left\|\partial_{k}\left|\nabla E_{j}\right|^{2}\right\|_{L^{2}} d s \\
& +C \int_{0}^{t} \sum_{j=1}^{N}\left\|\partial_{k}\left(\bar{E}_{j} \partial_{s} E_{j}\right)\right\|_{L^{2}} d s \\
& +C \int_{0}^{t}\left\|\partial_{k}\left(n|E|^{2}\right)\right\|_{L^{2}} d s \\
\leqq & C\left(\left\|\partial_{k} \nabla n_{0}\right\|_{L^{2}}+\left\|\partial_{k} n_{1}\right\|_{L^{2}}\right) \\
& +C \int_{0}^{t} \sum_{j=1}^{N}\left\|\nabla E_{j} \cdot \nabla \partial_{k} \bar{E}_{j}\right\|_{L^{2}} d s \\
& +C \int_{0}^{t} \sum_{j=1}^{N}\left(\left\|\left(\partial_{k} \bar{E}_{j}\right)\left(\partial_{s} E_{j}\right)\right\|_{L^{2}}+\left\|\bar{E}_{j} \partial_{k} \partial_{s} E_{j}\right\|_{L^{2}}\right) d s \\
& +C \int_{0}^{t}\left(\left\|\left(\partial_{k} n\right)|E|^{2}\right\|_{L^{2}}+\left\|n E \partial_{k} \bar{E}\right\|_{L^{2}}\right) d s, \\
& 0 \leqq t<\widetilde{T}_{m a x}, \quad 1 \leqq k \leqq N .
\end{aligned}
$$

By the definition of $\tilde{T}_{\max }$ we note that $\partial_{k} \nabla n, \partial_{k} \partial_{t} n \in L^{\infty}\left(0, T^{\prime} ; L^{2}\right)$ for any $T^{\prime}$ with $0<T^{\prime}<\widetilde{T}_{\max }$. By (3.64)-(3.66) and the Sobolev imbedding theorem we obtain

$$
\begin{aligned}
& \left\|\partial_{k} \nabla n(t)\right\|_{L^{2}}+\left\|\partial_{k} \partial_{t} n(t)\right\|_{L^{2}} \\
& \quad \leqq C+C \int_{0}^{t} \sum_{j=1}^{N}\left\|\partial_{k} E_{j}\right\|_{L^{4}}\left\|\partial_{k} \nabla E_{j}\right\|_{L^{4}} d s
\end{aligned}
$$




$$
\begin{aligned}
& +C \int_{0}^{t}\left(\left\|\partial_{k} E\right\|_{L^{4}}\left\|\partial_{s} E\right\|_{L^{4}}+\|E\|_{L^{4}}\left\|\partial_{k} \partial_{s} E\right\|_{L^{4}}\right) d s \\
& +C \int_{0}^{t}\left(\left\|\partial_{k} n\right\|_{L^{2}}\|E\|_{L^{\infty}}^{2}+\|n\|_{L^{4}}\|E\|_{L^{\infty}}\left\|\partial_{k} E\right\|_{L^{4}}\right) d s \\
& \leqq C+C \int_{0}^{t}\left\|\partial_{k} \partial_{s} E\right\|_{L^{4}} d s, \\
& \quad 0 \leqq t<\widetilde{T}_{\max }, \quad 1 \leqq k \leqq N .
\end{aligned}
$$

Inserting (3.71) into (3.67), we have

$$
\begin{aligned}
\left\|\partial_{k} \partial_{t} E(t)\right\|_{L^{4}} \leqq & \left\|U(t) \partial_{k} \partial_{t} E(0)\right\| L_{L^{4}} \\
& +C+C \int_{0}^{t}|t-s|^{-N / 4}\left\|\partial_{s} E\right\|_{L^{4}} d s \\
& +C \int_{0}^{t}|t-s|^{-N / 4}\left(C+C \int_{0}^{s}\left\|\partial_{k} \partial_{\tau} E\right\| L_{L^{4}} d \tau\right) d s \\
& +C \int_{0}^{t}|t-s|^{-N / 4}\left\|\partial_{k} \partial_{s} E\right\|_{L^{4}} d s \\
\leqq & \left\|U(t) \partial_{k} \partial_{t} E(0)\right\|_{L^{4}} \\
& +C+C \int_{0}^{t}|t-s|^{-N / 4}\left\|\partial_{s} E\right\|_{L^{4}} d s \\
& +C \int_{0}^{t}\left(|t-s|^{(4-N) / 4}+|t-s|^{-N / 4}\right)\left\|\partial_{k} \partial_{s} E\right\|_{L^{4}} d s, \\
& \quad 0 \leqq t<\widetilde{T}_{\text {max }}, \quad 1 \leqq k \leqq N .
\end{aligned}
$$

Here at the second inequality of (3.72) we have used the following identity:

$$
\begin{aligned}
\int_{0}^{t}|t-s|^{-N / 4} \int_{0}^{s} & \left\|\partial_{k} \partial_{\tau} E\right\|_{L^{4}} d \tau d s \\
& =(1-N / 4)^{-1} \int_{0}^{t}|t-s|^{(4-N) / 4}\left\|\partial_{k} \partial_{s} E\right\|_{L^{4}} d s .
\end{aligned}
$$

Lemma 2.1(ii) with $q=4$ implies that the first term at the right hand side of (3.72) belongs to $L^{8 / N}(\boldsymbol{R})$, and (3.66) and the Hardy-Littlewood-Sobolev inequality imply that the second term at the right hand side of (3.72) belongs to $L^{\infty}\left(0, \widetilde{T}_{\max }\right)$ for $N=1,2$ and to $L^{8}\left(0, \widetilde{T}_{\max }\right)$ for $N=3$. Therefore, (3.72) and the theory of the Volterra type integral equation yield

$$
\partial_{k} \partial_{t} E \in L^{8 / N}\left(0, \widetilde{T}_{\max } ; L^{4}\right), \quad 1 \leqq k \leqq N .
$$

We next take the $L^{\infty}\left(0, \widetilde{T}_{\text {max }} ; L^{2}\right)$ norm of (3.62) and use Lemma 2.2 with $q^{\prime}=4 / 3$ and $r^{\prime}=8 /(8-N)$ to obtain by (3.64)-(3.66) and (3.71) 


$$
\begin{aligned}
& \left\|\partial_{k} \partial_{t} E\right\|_{L^{\infty}\left(0, \widetilde{T}_{m a x} ; L^{2}\right)} \\
& \quad \leqq C+C\left\|\partial_{k} \partial_{t} E\right\|_{L^{8 / N}\left(0, \widetilde{T}_{m a x} ; L^{4}\right), \quad 1 \leqq k \leqq N}
\end{aligned}
$$

in the same way as (3.72) . (3.74) and (3.73) show that

$$
\partial_{k} \partial_{t} E \in L^{\infty}\left(0, \widetilde{T}_{m a x} ; L^{2}\right), \quad 1 \leqq k \leqq N .
$$

(3.64)-(3.66) and the Sobolev imbedding theorem imply that the right hand side of (1.1) belongs to $L^{\infty}\left(0, T ; H^{1}\right)$. Accordingly, (3.75), (3.65), (1.1) and the regularity theorem of the elliptic equation give us

$$
E \in L^{\infty}\left(0, \widetilde{T}_{\max } ; H^{3}\right) \text {. }
$$

(3.76) and (1.2) imply that

$$
\begin{aligned}
& n \in L^{\infty}\left(0, \widetilde{T}_{m a x} ; H^{2}\right), \\
& \partial_{t} n \in L^{\infty}\left(0, \widetilde{T}_{m a x} ; H^{1}\right) .
\end{aligned}
$$

(3.76)-(3.78) assure that by Proposition 3.2 we can extend the solutions $(E(t)$, $n(t))$ as the solutions in $H^{3} \oplus H^{2}$ beyond $t=\widetilde{T}_{\text {max }}$. This contradicts the definition of $\tilde{T}_{m a x}$.

Thus, the proof for even $m$ is complete. In the same way as above we can prove Proposition 3.3 for odd $m$.

By combining Propositions 3.1, 3.3 and the induction argument we obtain Theorem 1.1(2)-(3). Thus, the proof of Theorem 1.1 is completed.

We conclude this section by giving the following theorem concerning the existence of global solutions for (1.1)-(1.3).

Theorem 3.4. (1) Assume $N=1$. Let $m$ be an integer with $m \geqq 2$. If $\left(E_{0}, n_{0}, n_{1}\right) \in H^{m} \oplus H^{m-1} \oplus H^{m-2}$ and $n_{1} \in \dot{H}^{-1}$, then the existence time $T$ of the solutions in Theorem 1.1 can be chosen as $T=+\infty$. Furthermore, if $E_{0}, n_{0}, n_{1} \in$ $\bigcap_{m=1}^{\infty} H^{m}$ and $n_{1} \in \dot{H}^{-1}$, then the solutions $E(t, x)$ and $n(t, x)$ are in $C^{\infty}([0, \infty) \times \mathbb{R})$. (2) Assume $N=2$. Let $m$ be an integer with $m \geqq 2$. There exists $\delta>0$ such that if $\left(E_{0}, n_{0}, n_{1}\right) \in H^{m} \oplus H^{m-1} \oplus H^{m-2}, n_{1} \in \dot{H}^{-1}$ and $\left\|E_{0}\right\|_{L^{2}<\delta}$, then the existence time $T$ of the solutions in Theorem 1.1 can be chosen as $T=+\infty$. In addition, if $E_{0}, n_{0}$, and $n_{1}$ are in $\bigcap_{m=1}^{\infty} H^{m}$, then the solutions $E(t, x)$ and $n(t, x)$ are in $C^{\infty}\left([0, \infty) \times \boldsymbol{R}^{2}\right)$.

The a priori estimates needed for the proof of existence of global solutions are already established by C. Sulem and P.L. Sulem [17, Proof of Theorme 2] 
and by H. Added and S. Added [1, Proof of Theorem] (see also [12]). The proof of the a priori estimates requires the assumption $n_{1} \in \dot{H}^{-1}$, because the energy identity of (1.1)-(1.3) contains the $\dot{H}^{-1}$ norm of $n_{1}$. Those a priori estimates and Theorem 1.1 show Theorem 3.4.

\section{§4. Proof of Theorem 1.2}

In this section we give the proof of Theorem 1.2. We first describe the proof of Part (1) of Theorem 1.2.

Proof of Theorem 1.2(1). Let $(E(t), n(t))$ be the solutions of (1.1)-(1.3) in the class of Theorem 1.1.

We first assume that $m$ is even. Then we can easily see by (1.1) that $E(t) \in$ $C^{m / 2+1}\left(\left[0, T_{m a x}\right) ; H^{-2}\right)$. We differentiate (1.1) $m / 2$ times in $t$ to obtain

$$
\begin{gathered}
i \partial_{t}\left(\partial_{t}^{m / 2} E\right)+\Delta\left(\partial_{t}^{m / 2} E\right)=\sum_{j=0}^{m / 2}\left(\begin{array}{c}
m / 2 \\
j
\end{array}\right)\left(\partial_{t}^{m / 2-j} n\right)\left(\partial_{t}^{j} E\right) \\
\text { in } H^{-2}, \quad 0 \leqq t<T_{m a x} .
\end{gathered}
$$

We rewrite (4.1) as the integral form:

$$
\begin{gathered}
\partial_{t}^{m / 2} E(t)=U(t) \partial_{t}^{m / 2} E(0) \\
-i \int_{0}^{t} U(t-s) \sum_{j=0}^{m / 2}\left(\begin{array}{c}
m / 2 \\
j
\end{array}\right)\left(\partial_{s}^{m / 2-j} n\right)\left(\partial_{s}^{j} E\right) d s, \\
0 \leqq t<T_{\max } .
\end{gathered}
$$

Since $(E(t), n(t))$ are in the class of Theorem 1.1, we have

$$
\begin{aligned}
& E \in \bigcap_{j=0}^{m / 2} C^{j}\left(\left[0, T_{\text {max }}\right) ; H^{m-2 j}\right), \\
& E \in \bigcap_{j=0}^{m / 2} W^{j, 8 / N}\left(0, T ; W^{m-2 j, 4}\right), \quad 0<T<T_{\max }, \\
& n \in \bigcap_{j=0}^{3} C^{j}\left(\left[0, T_{\text {max }}\right) ; H^{m-1-j}\right),
\end{aligned}
$$

and if $m \geqq 6$.

$$
n \in \bigcap_{j=4}^{m / 2+1} C^{j}\left(\left[0, T_{\max }\right) ; H^{m+2-2 j}\right) .
$$

On the other hand, by the Sobolev imbedding theorem we have 


$$
\begin{aligned}
\left\|\sum_{j=0}^{m / 2}\left(\begin{array}{c}
m / 2 \\
j
\end{array}\right)\left(\partial_{s}^{m / 2-j} n\right)\left(\partial_{s}^{j} E\right)\right\|_{L^{2}} \leqq & C \sum_{j=0}^{m / 2-1}\left\|\partial_{s}^{m / 2-j} n\right\|_{L^{2}}\left\|\partial_{s}^{j} E\right\|_{L^{\infty}} \\
& +\|n\|_{L^{4}}\left\|\partial_{s}^{m / 2} E\right\|_{L^{4}} \\
\leqq & C \sum_{j=0}^{m / 2-1}\left\|\partial_{s}^{m / 2-j} n\right\|_{L^{2}}\left\|\partial_{s}^{j} E\right\|_{H^{2}} \\
& +C\|n\|_{H^{1}}\left\|\partial_{s}^{m / 2} E\right\|_{L^{4}} .
\end{aligned}
$$

(4.3)-(4.7) give us

$$
\sum_{j=0}^{m / 2}\left(\begin{array}{c}
m / 2 \\
j
\end{array}\right)\left(\partial_{s}^{m / 2-j} n\right)\left(\partial_{s}^{j} E\right) \in L^{8 / N}\left(0, T ; L^{2}\right), \quad 0<T<T_{m a x} .
$$

Noting $\partial_{\varepsilon}^{m / 2} E(0) \in L^{2}$, we apply Lemma 2.3 to (4.2) and use (4.8) to obtain

$$
\begin{aligned}
\partial_{t}^{m / 2} E \in L^{2}(0, T ; & \left.H^{1 / 2}(|x|<R)\right), \\
& 0<T<T_{\max x}, \quad R>0 .
\end{aligned}
$$

We differentiate (1.1) $m / 2-1$ times in $t$ to obtain

$$
\begin{gathered}
i \partial_{t}\left(\partial_{t}^{m / 2-1} E\right)+\Delta\left(\partial_{t}^{m / 2-1} E\right) \\
=\sum_{j=0}^{m / 2-1}\left(\begin{array}{c}
m / 2-1 \\
j
\end{array}\right)\left(\partial_{t}^{m / 2-1-j} n\right)\left(\partial_{\varepsilon}^{j} E\right), \\
0 \leqq t<T_{\max } .
\end{gathered}
$$

(4.3), (4.5), (4.6) and the Sobolev imbedding theorem imply that the right hand side of (4.10) is in $L^{\infty}\left(0, T ; H^{1}\right)$ for $0<T<T_{m a x}$. Therefore, (4.9), (4.10) and the local regularity theorem of the elliptic equation yield

$$
\partial_{\delta}^{m / 2-1} E \in L^{2}\left(0, T ; H^{5 / 2}(|x|<R)\right), \quad 0<T<T_{m a x}, \quad R>0 .
$$

If $m / 2-1<0$,we repeat the same argument as above until we have

$$
E \in L^{2}\left(0, T ; H^{m+1 / 2}(|x|<R)\right), \quad 0<T<T_{m a x}, \quad R>0 .
$$

Thus, the proof for even $m$ is completed. We similarly prove Theorem 1.2(1) for $m$ odd. 閳

We next state the proof of Part (2) of Theorem 1.2.

Proof of Theorem 1.2(2). We prove Theorem 1.2(2) only in the case where $m$ is an even integer with $m \geqq 6$ and $k=2$, since the proof for the other cases is the same.

Let $(E(t), n(t))$ be the solutions of (1.1)-(1.3) in the class of Theorem 1.2(2) for any $T$ with $0<T<T_{m a x}$. Let $T$ be an arbitrarily fixed constant with 
$0<T<T_{\max }$. We put $n_{\mathrm{g}}(t)=\rho_{\mathrm{g}} * n(t)$ and put

$$
A(n)=\sum_{j=0}^{3}\left\|\partial_{t}^{j} n\right\|_{L^{\infty}\left(0, T ; H^{m-1-j}\right)}+\sum_{j=4}^{m / 2+1}\left\|\partial_{t}^{j} n\right\|_{L^{\infty}\left(0, T ; H^{m+2-2 j}\right)}
$$

We note that $n_{\mathrm{g}}(t) \in C^{m / 2+1}\left([0, T] ; H^{s}\right)$ for $s>0$ and $A\left(n_{\mathrm{\varepsilon}}\right) \leqq A(n)$. Let $E_{0 \mathrm{\varepsilon}} \in \mathcal{S}$ such that $E_{0 \varepsilon} \rightarrow E_{0}$ in $H^{m, 2}$ as $\varepsilon \rightarrow 0$ and $\left\|E_{0 \varepsilon}\right\|_{H^{m, 2}} \leqq 2\left\|E_{0}\right\|_{H^{m, 2}}$. We consider the following linear Schrödinger equation:

$$
\begin{aligned}
& i \partial_{t} E_{\mathrm{q}}+\Delta E_{\mathrm{g}}=n_{\mathrm{q}} E_{\mathrm{q}}, \quad 0 \leqq t \leqq T, \quad x \in \boldsymbol{R}^{N}, \\
& E_{\mathrm{q}}(0, x)=E_{0 \mathrm{~g}}(x) .
\end{aligned}
$$

By the theory of evolution equation we have the unique solution $E_{\mathrm{g}}(t)$ of (4.13) and (4.14) such that $E_{\mathrm{\varepsilon}} \in C^{m / 2+1}\left([0, T] ; H^{s}\right)$ for $s>0$ and

$$
\left\|\partial_{i}^{j} E_{\varepsilon}\right\|_{L^{\infty}\left(0, T ; H^{m-2 j}\right)} \leqq C_{1}, \quad 0 \leqq j \leqq m / 2,
$$

where $C_{1}$ depends only on $T, A(n),\left\|E_{0}\right\|_{H^{m}}$ and $N$ but not on $\varepsilon$. We put $g_{l}(x)=$ $\left(1+|x / l|^{2}\right)^{-2}$ for a positive integer $l$. A simple calculation gives us

$$
\begin{aligned}
& \left|\nabla\left\{\left(1+|x|^{2}\right) g_{l}^{2}(x)\right\}\right| \leqq C_{2}\left(1+|x|^{2}\right)^{1 / 2} g_{l}^{2}(x), \\
& \left|\nabla\left\{\left(1+|x|^{2}\right)^{2} g_{l}^{2}(x)\right\}\right| \leqq C_{3}\left(1+|x|^{2}\right)^{3 / 2} g_{l}^{2}(x)
\end{aligned}
$$

where $C_{2}$ and $C_{3}$ do not depend on $l$.

We consider the scalar product in $L^{2}$ between (4.13) and $\left(1+|x|^{2}\right) g_{l}^{2}(x) E_{\varepsilon}(t)$ and take the imaginary part of the resulting equation to obtain by (4.16)

$$
\begin{aligned}
& \left\|\left(1+|x|^{2}\right)^{1 / 2} g_{l} E_{\mathrm{\varepsilon}}(t)\right\|_{L^{2}}^{2} \leqq\left\|\left(1+|x|^{2}\right)^{1 / 2} g_{l} E_{0 \mathrm{e}}\right\|_{L^{2}}^{2} \\
& +C \int_{0}^{t} \sum_{j=1}^{N}\left\|g_{l} \nabla E_{\mathrm{z} j}\right\|_{L^{2}}\left\|\left(1+|x|^{2}\right)^{1 / 2} g_{l} E_{\mathrm{e}}\right\|_{L^{2}} d s \\
& \leqq\left\|E_{0}\right\|_{H^{m, 2}}^{2} \\
& +C \int_{0}^{t} \sum_{j=1}^{N}\left\|\nabla E_{\mathrm{q} j}\right\|_{L^{2}}\left\|\left(1+|x|^{2}\right)^{1 / 2} g_{l} E_{\mathrm{q}}\right\|_{L^{2}} d s, \\
& 0 \leqq t \leqq T \text {. }
\end{aligned}
$$

(4.15), (4.18) and Gronwall's inequality yield

$$
\left\|\left(1+|x|^{2}\right)^{1 / 2} g_{l} E_{\mathrm{q}}\right\|_{L^{\infty}\left(0, T ; L^{2}\right)} \leqq C_{4},
$$

where $C_{4}$ depends only on $T,\left\|E_{0}\right\|_{H^{m, 2}}, A(n)$ and $N$ but not on $l$ and $\varepsilon$. Letting $l \rightarrow \infty$ in (4.19), by Fatou's lemma we obtain 


$$
\left\|\left(1+|x|^{2}\right)^{1 / 2} E_{\mathrm{e}}\right\|_{L^{\infty}\left(0, T ; L^{2}\right) \leqq C_{4}} .
$$

We next differentiate (4.13) in $x_{k}, 1 \leqq k \leqq N$ and take the imaginary part of the scalar product in $L^{2}$ between the resulting equtaion and $\left(1+|x|^{2}\right) g_{l}^{2}(x) \partial_{k} E_{\mathrm{g}}(t)$ to obtain by $(4.18)$

$$
\begin{aligned}
& \left\|\left(1+|x|^{2}\right)^{1 / 2} g_{l} \partial_{k} E_{\mathrm{g}}(t)\right\|_{L^{2}}^{2} \leqq\left\|\left(1+|x|^{2}\right)^{1 / 2} g_{l} \partial_{k} E_{0 \mathrm{e}}\right\|_{L^{2}}^{2} \\
& +C \int_{0}^{t}\left[\sum_{j=1}^{J}\left\|g_{l} \partial_{k} \nabla E_{\mathrm{z} j}\right\|_{L^{2}}\left\|\left(1+|x|^{2}\right)^{1 / 2} g_{l} \partial_{k} E_{\mathrm{e}}\right\|_{L^{2}}\right. \\
& \left.+\left\|\partial_{k} n_{\mathrm{e}}\right\|_{L^{\infty}}\left\|\left(1+|x|^{2}\right)^{1 / 2} g_{l} E_{\mathrm{q}}\right\|_{L^{2}}\left\|\left(1+|x|^{2}\right)^{1 / 2} g_{l} \partial_{k} E_{\mathrm{e}}\right\|_{L^{2}}\right] d s \\
& \leqq C|| E_{0} \|_{H^{m, 2}} \\
& +C \int_{0}^{t}\left[\sum_{j=0}^{N}\left\|\partial_{k} \nabla E_{\mathrm{\varepsilon}}\right\|_{L^{2}}\right. \\
& \left.+\left\|\partial_{k} n_{\mathrm{\varepsilon}}\right\|_{L^{\infty}}\left\|\left(1+|x|^{2}\right)^{1 / 2} g_{l} E_{\mathrm{\varepsilon}}\right\|_{L^{2}}\right]\left\|\left(1+|x|^{2}\right)^{1 / 2} g_{l} \partial_{k} E_{\mathrm{\varepsilon}}\right\|_{L^{2}} d s, \\
& 0 \leqq t \leqq T, \quad 1 \leqq k \leqq N .
\end{aligned}
$$

(4.15), (4.21), the Sobolev imbedding theorem and Gronwall's inequality yield

$$
\left\|\left(1+|x|^{2}\right)^{1 / 2} g_{l} \partial_{k} E_{\mathrm{e}}\right\|_{L^{\infty}\left(0, T ; L^{2}\right)} \leqq C_{5}, \quad 1 \leqq k \leqq N,
$$

where $C_{5}$ depends only on $T,\left\|E_{0}\right\|_{H^{m, 2}, A(n)}$ and $N$ but not on $l$ and $\varepsilon$. Letting $l \rightarrow \infty$ in (4.22), by Fatou's lemma we obtain

$$
\left\|\left(1+|x|^{2}\right)^{1 / 2} \partial_{k} E_{\mathrm{\varepsilon}}\right\|_{L^{\infty}\left(0, T ; L^{2}\right)} \leqq C_{5}, \quad 1 \leqq k \leqq N .
$$

We take the imaginary part of the scalar product in $L^{2}$ between (4.13) and (1+ $\left.|x|^{2}\right)^{2} g_{l}^{2} E_{\mathrm{g}}(t)$ to obtain by (4.17)

$$
\begin{aligned}
& \left\|\left(1+|x|^{2}\right) E_{\mathrm{g}}(t)\right\|_{L^{2}}^{2} \leqq\left\|\left(1+|x|^{2}\right) g_{l} E_{0 \mathrm{e}}\right\|_{L^{2}}^{2} \\
& +C \int_{0}^{t} \sum_{j=1}^{N}\left\|(1+|x|)^{1 / 2} g_{l} \nabla E_{\mathrm{e} j}\right\| L_{L^{2}} \mid\left\|\left(1+|x|^{2}\right) g_{l} E_{\mathrm{e}}\right\|_{L^{2}} d s \\
& \leqq C\left\|E_{0}\right\|_{H^{m, 2}}^{2} \\
& +C \int_{0}^{t} \sum_{j=1}^{N}\left\|\left(1+|x|^{2}\right)^{1 / 2} \nabla E_{g_{j}}\right\|_{L^{2}}\left\|\left(1+|x|^{2}\right) g_{l} E_{\mathrm{q}}\right\|_{L^{2}} d s, \\
& 0 \leqq t \leqq T .
\end{aligned}
$$

(4.23), (4.24) and Gronwall's inequality yield

$$
\left\|\left(1+|x|^{2}\right) g_{l} E_{\mathrm{\varepsilon}}\right\|_{L^{\infty}\left(0, T ; L^{2}\right)} \leqq C_{6},
$$

where $C_{6}$ depends only on $T,\left\|E_{0}\right\|_{H^{m, 2}}, A(n)$ and $N$ but not on $l$ and $\varepsilon_{\text {. }}$ Letting $l \rightarrow \infty$ in (4.25), by Fatou's lemma we have 


$$
\left\|\left(1+|x|^{2}\right) E_{\mathrm{g}}\right\|_{L^{\infty}\left(0, T ; L^{2}\right)} \leqq C_{6} .
$$

We next show that if

$$
\begin{aligned}
& \left\|\left(1+|x|^{2}\right) \partial_{\xi}^{p} E_{\mathrm{q}}\right\|_{L^{\infty}\left(0, T ; L^{2}\right)} \leqq C(q), \quad 0 \leqq p \leqq q, \\
& \left\|\left(1+|x|^{2}\right)^{1 / 2} \partial_{k} \partial_{\varepsilon}^{p} E_{\mathrm{q}}\right\|_{L^{\infty}\left(0, T ; L^{2}\right)} C \leqq(q), \quad 1 \leqq k \leqq N, \quad 0 \leqq p \leqq q
\end{aligned}
$$

for some integer $q$ with $0 \leqq q \leqq m / 2-2$, then (4.27) and (4.28) also hold with $q$ and $C(q)$ replaced by $q+1$ and $C(q+1)$, where $C(q)$ depends only on $T,\left\|E_{0}\right\|_{H^{m, 2} \text {, }}$ $A(n), N$ and $q$ but not on $\varepsilon$. We differentiate (4.13) $q+1$ times in $t$ and take the imaginary part of the scalar product in $L^{2}$ between the resulting equation and $\left(1+|x|^{2}\right) g_{l}^{2} \partial_{t}^{q+1} E_{\mathrm{e}}(t)$ to obtain by (4.16)

$$
\begin{aligned}
& \left\|\left(1+|x|^{2}\right)^{1 / 2} g_{l} \partial_{\mathrm{g}}^{q+1} E_{\mathrm{g}}(t)\right\|_{L^{2}}^{2} \leqq\left\|\left(1+|x|^{2}\right)^{1 / 2} g_{l} \partial_{l}^{q+1} E_{\mathrm{g}}(0)\right\|_{L^{2}} \\
& +C \int_{0}^{t} \sum_{j=1}^{N T}\left\|g_{l} \nabla \partial_{s}^{q+1} E_{\mathrm{g} j}\right\|_{L^{2}}\left\|\left(1+|x|^{2}\right)^{1 / 2} g_{l} \partial_{s}^{q+1} E_{\mathrm{g}}\right\|_{L^{2}} d s \\
& +C \int_{0}^{t} \sum_{j=0}^{q}\left\|\partial_{s}^{q+1-j} n_{\mathrm{g}}\right\|_{L^{\infty}}\left\|\left(1+|x|^{2}\right)^{1 / 2} g_{l} \partial_{s}^{j} E_{\mathrm{q}}\right\|_{L^{2}} \\
& \quad \times\left\|\left(1+|x|^{2}\right)^{1 / 2} g_{l} \partial_{s}^{q+1} E_{\mathrm{\varepsilon}}\right\|_{L^{2}} d s \\
& \leqq C+C \int_{0}^{t}\left[\sum_{j=1}^{N}\left\|\nabla \partial_{s}^{q+1} E_{\mathrm{g}}\right\|_{L^{2}}\right. \\
& \left.\quad+\sum_{j=0}^{q}\left\|\partial_{s}^{q+1-j} n_{\mathrm{\varepsilon}}\right\|_{L^{\infty}}\left\|\left(1+|x|^{2}\right)^{1 / 2} g_{l} \partial_{s}^{j} E_{\mathrm{\varepsilon}}\right\|_{L^{2}}\right] \\
& \quad \times\left\|\left(1+|x|^{2}\right)^{1 / 2} g_{l} \partial_{s}^{q+1} E_{\mathrm{\varepsilon}}\right\|_{L^{2}} d s .
\end{aligned}
$$

(4.15), (4.29), the Sobolev imbedding theorem and Gronwall's inequality yield

$$
\left\|\left(1+|x|^{2}\right)^{1 / 2} g_{l} \partial_{\mathrm{g}}^{q+1} E_{\mathrm{e}}\right\|_{L^{\infty}\left(0, T ; L^{2}\right)} \leqq C_{7},
$$

where $C_{7}$ depends only on $T,\left\|E_{0}\right\|_{H^{m, 2}}, A(n)$ and $N$ but not on $l$ and $\varepsilon$. Letting $l \rightarrow \infty$ in (4.30), by Fatou's lemma we obtain

$$
\left\|\left(1+|x|^{2}\right)^{1 / 2} \partial_{t}^{q+1} E_{\mathrm{g}}\right\|_{L^{\infty}\left(0, T ; L^{2}\right)} \leqq C_{7} .
$$

We next differentiate (4.13) $q+1$ times in $t$ and once in $x_{k}, 1 \leqq k \leqq N$, and take the imaginary part of the scalar product in $L^{2}$ between the resulting equation and $\left(1+|x|^{2}\right) g_{l}^{2} \partial_{k} \partial_{t}^{q+1} E_{\mathrm{g}}(t)$ to obtain by (4.16)

$$
\begin{aligned}
\left\|\left(1+|x|^{2}\right)^{1 / 2} g_{l} \partial_{k} \partial_{t}^{q+1} E_{\mathrm{g}}(t)\right\|_{L^{2}}^{2} \leqq\left\|\left(1+|x|^{2}\right)^{1 / 2} g_{l} \partial_{k} \partial_{t}^{q+1} E_{\mathrm{g}}(0)\right\|_{L^{2}}^{2} \\
\quad \dot{+} C \int_{0}^{t} \sum_{j=1}^{N}\left\|g_{l} \partial_{k} \nabla \partial_{s}^{q+1} E_{\mathrm{g} j}\right\|\left\|_{L^{2}}\right\|\left(1+|x|^{2}\right)^{1 / 2} g_{l} \partial_{k} \partial_{s}^{q+1} E_{\mathrm{g}} \|_{L^{2}} d s
\end{aligned}
$$




$$
\begin{aligned}
+C \int_{0}^{t}\left[\sum_{j=0}^{q+1}\left\|\partial_{k} \partial_{s}^{q+1-j} n_{\mathrm{g}}\right\|_{L^{\infty}}\left\|\left(1+|x|^{2}\right)^{1 / 2} g_{l} \partial_{s}^{j} E_{\mathrm{e}}\right\|_{L^{2}}\right. \\
\left.+\sum_{j=0}^{q}\left\|\partial_{s}^{q+1-j} n_{\mathrm{e}}\right\|_{L^{\infty}}\left\|\left(1+|x|^{2}\right)^{1 / 2} g_{l} \partial_{k} \partial_{s}^{j} E_{\mathrm{\varepsilon}}\right\|_{L^{2}}\right] \\
\quad \times\left\|\left(1+|x|^{2}\right)^{1 / 2} g_{l} \partial_{k} \partial_{s}^{q+1} E_{\mathrm{\varepsilon}}\right\|_{L^{2}} d s \\
\leqq C+C \int_{0}^{t}\left[\sum_{j=1}^{J}\left\|\partial_{k} \nabla \partial_{s}^{q+1} E_{\mathrm{\varepsilon} j}\right\|_{L^{2}}\right. \\
+\sum_{j=0}^{q+1}\left\|\partial_{k} \partial_{s}^{q+1-j} n_{\mathrm{e}}\right\|_{L^{\infty}}\left\|\left(1+|x|^{2}\right)^{1 / 2} \partial_{s}^{j} E_{\mathrm{e}}\right\|_{L^{2}} \\
\left.+\sum_{j=0}^{q}\left\|\partial_{s}^{q+1-j} n_{\mathrm{\varepsilon}}\right\|_{L^{\infty}}\left\|\left(1+|x|^{2}\right)^{1 / 2} \partial_{k} \partial_{s}^{j} E_{\mathrm{\varepsilon}}\right\|_{L^{2}}\right] \\
\quad \times\left\|\left(1+|x|^{2}\right)^{1 / 2} g_{l} \partial_{k} \partial_{s}^{q+1} E_{\mathrm{\varepsilon}}\right\|_{L^{2}} d s, \\
\quad 0 \leqq t \leqq T, \quad 1 \leqq k \leqq N .
\end{aligned}
$$

(4.15), (4.27), (4.28), (4.31), (4.32), the Sobolev imbedding theorem and Gronwall's inequality yield

$$
\left\|\left(1+|x|^{2}\right)^{1 / 2} g_{l} \partial_{k} \partial_{t}^{q+1} E_{\mathrm{g}}\right\|_{L^{\infty}\left(0, T ; L^{2}\right)} \leqq C_{8}, \quad 1 \leqq k \leqq N,
$$

where $C_{8}$ depends only on $T,\left\|E_{0}\right\|_{H^{m, 2}}, A(n)$ and $N$ but not on $l$ and $\varepsilon_{\text {. Letting }}$ $l \rightarrow \infty$ in (4.33), by Fatou's lemma we obtain

$$
\left\|\left(1+|x|^{2}\right)^{1 / 2} \partial_{t}^{q+1} E_{\varepsilon}\right\|_{L^{\infty}\left(0, T ; L^{2}\right)} \leqq C_{8}, \quad 1 \leqq k \leqq N .
$$

We differentiate (4.13) $q+1$ times in $t$ again and take the imaginary part of the scalar product in $L^{2}$ between the resulting equation and $\left(1+|x|^{2}\right)^{2} g_{l} E_{\varepsilon}(t)$ to obtain by (4.17)

$$
\begin{aligned}
& \left\|\left(1+|x|^{2}\right) g_{l} \partial_{t}^{q+1} E_{\mathrm{g}}(t)\right\|_{L}^{2} \leqq\left\|\left(1+|x|^{2}\right) g_{l} \partial_{z}^{q+1} E_{\mathrm{e}}(0)\right\|_{L^{2}}^{2} \\
& +C \int_{0}^{t} \sum_{j=1}^{N}\left\|\left(1+|x|^{2}\right)^{1 / 2} g_{l} \nabla \partial_{s}^{q+1} E_{\mathrm{q} j}\right\|_{L^{2}}\left\|\left(1+|x|^{2}\right) g_{l} \partial_{s}^{q+1} E_{\mathrm{q}}\right\|_{L^{2}} d s \\
& +C \int_{0}^{t} \sum_{j=0}^{q+1}\left\|\partial_{s}^{q+1-j} n_{\mathrm{\varepsilon}}\right\|_{L^{\infty}}\left\|\left(1+|x|^{2}\right) g_{l} \partial_{s}^{j} E_{\mathrm{\varepsilon}}\right\|_{L^{2}} \\
& \times\left\|\left(1+|x|^{2}\right) g_{l} \partial_{s}^{q+1} E_{\mathrm{\varepsilon}}\right\|_{L^{2}} d s \\
& \leqq C+C \int_{0}^{t}\left[\sum_{j=1}^{N}\left\|\left(1+|x|^{2}\right)^{1 / 2} \nabla \partial_{s}^{q+1} E_{\varepsilon_{j}}\right\|_{L^{2}}\right. \\
& \left.+\sum_{j=0}^{q}\left\|\partial_{s}^{q+1-j} n_{\mathrm{\varepsilon}}\right\|_{L^{\infty}}\left\|\left(1+|x|^{2}\right) \partial_{s}^{j} E_{\mathrm{\varepsilon}}\right\|_{L^{2}}\right] \\
& \times\left\|\left(1+|x|^{2}\right) g_{l} \partial_{s}^{q+1} E_{\mathrm{\varepsilon}}\right\|_{L^{2}} d s, \\
& 0 \leqq t \leqq T \text {. }
\end{aligned}
$$


(4.15), (4.27), (4.28), (4.34), the Sobolev imbedding theorem and Gronwall's inequality yield

$$
\left\|\left(1+|x|^{2}\right) g_{l} \partial_{t}^{q+1} E_{\mathrm{e}}\right\|_{L^{\infty}\left(0, T ; L^{2}\right)} \leqq C_{9},
$$

where $C_{9}$ depends only on $T,\left\|E_{0}\right\|_{H^{m, 2}, A(n)}$ and $N$ but not on $l$ and $\varepsilon$. Letting $l \rightarrow \infty$ in (4.36) by Fatou's lemma we obtain

$$
\left\|\left(1+|x|^{2}\right) \partial_{t}^{q+1} E_{\mathrm{q}}\right\|_{L^{\infty}\left(0, T ; L^{2}\right)} \leqq C_{9} .
$$

Hence, (4.23), (4.26) and the induction argument imply that

(4.38) $\quad\left\|\left(1+|x|^{2}\right) \partial_{t}^{j} E_{\mathrm{q}}\right\|_{L^{\infty}\left(0, T ; L^{2}\right)} \leqq C_{10}, \quad 0 \leqq j \leqq m / 2-j$,

(4.39) $\left\|\left(1+|x|^{2}\right)^{1 / 2} \partial_{k} \partial_{t}^{j} E_{\mathrm{e}}\right\|_{L^{\infty}\left(0, T ; L^{2}\right)} \leqq C_{11}, \quad 0 \leqq j \leqq m / 2-1, \quad 1 \leqq k \leqq N$,

where $C_{10}$ and $C_{11}$ depend only on $T,\left\|E_{0}\right\|_{H^{m, 2}}, A(n)$ and $N$ but not on $\varepsilon$.

Noting that $E_{0 \mathrm{e}} \in \mathcal{S}$ and $n_{\mathrm{\varepsilon}} \in C^{m / 2+1}\left([0, T] ; H^{s}\right)$ for $s>0$, we can similarly show that $\left(1+|x|^{2}\right) \partial_{t}^{m / 2} E \in L^{\infty}\left(0, T ; L^{2}\right)$ and $\left(1+|x|^{2}\right)^{1 / 2} \partial_{k} \partial_{t}^{m / 2} E \in$ $L^{\infty}\left(0, T ; L^{2}\right), 1 \leqq k \leqq N$. We differentiate (4.13) $m / 2$ times in $t$ to obtain

$$
\begin{aligned}
& i \partial_{t}\left(\partial_{t}^{m / 2} E_{\mathrm{g}}\right)+\Delta\left(\partial_{t}^{m / 2} E_{\mathrm{\varepsilon}}\right) \\
& \quad=\sum_{j=0}^{m / 2}\left(\begin{array}{c}
m / 2 \\
j
\end{array}\right)\left(\partial_{t}^{m / 2-j} n_{\mathrm{g}}\right)\left(\partial_{\mathrm{t}}^{j} E_{\mathrm{g}}\right), \quad 0 \leqq t \leqq T .
\end{aligned}
$$

We rewrite (4.40) as the integral form:

$$
\begin{gathered}
\partial_{t}^{m / 2} E_{\mathrm{q}}(t)=U(t) \partial_{t}^{m / 2} E_{\mathrm{\varepsilon}}(0) \\
-i \int_{0}^{t} U(t-s) \sum_{j=0}^{m / 2}\left(\begin{array}{c}
m / 2 \\
j
\end{array}\right)\left(\partial_{s}^{m / 2-j} n_{\mathrm{\varepsilon}}\right)\left(\partial_{s}^{j} E_{\mathrm{q}}\right) d s, \\
0 \leqq t \leqq T .
\end{gathered}
$$

By (4.41) and Lemma 2.4 we obtain

$$
\begin{gathered}
J_{k}^{2}(t) \partial_{t}^{m / 2} E_{\mathrm{g}}(t)=U(t) x_{k}^{2} \partial_{t}^{m / 2} E_{\mathrm{g}}(0) \\
-i \int_{0}^{t} U(t-s) \sum_{j=0}^{m / 2}\left(\begin{array}{c}
m / 2 \\
j
\end{array}\right) J_{k}^{2}(s)\left\{\left(\partial_{s}^{m / 2-j} n_{\mathrm{g}}\right)\left(\partial_{s}^{j} E_{\mathrm{q}}\right)\right\} d s, \\
0 \leqq t \leqq T, \quad 1 \leqq k \leqq N .
\end{gathered}
$$

We take the $L^{2}$ norm of (4.42) to obtain

$$
\begin{aligned}
& \left\|J_{k}^{2} \partial_{t}^{m / 2} E_{\mathrm{q}}(t)\right\|_{L^{2}} \leqq\left\|x_{k}^{2} \partial_{t}^{m / 2} E_{\mathrm{q}}(0)\right\|_{L^{2}} \\
& \quad+C \int_{0}^{t} \sum_{j=0}^{m / 2}\left\|M(s)\left(2 i s \partial_{k}\right)^{2}\left\{M(-s)\left(\partial_{s}^{m / 2-j} n_{\mathrm{\varepsilon}}\right)\left(\partial_{s}^{j} E_{\mathrm{g}}\right)\right\}\right\|_{L^{2}} d s
\end{aligned}
$$




$$
\begin{aligned}
& \leqq C+C \int_{0}^{t} s^{2} \sum_{j=0}^{m / 2}\left[\left\|\left(\partial_{k}^{2} \partial_{s}^{m / 2-j} n_{\varepsilon}\right)\left(\partial_{s}^{j} E_{\varepsilon}\right)\right\|_{L^{2}}\right. \\
& +\left\|\left(\partial_{k} \partial_{s}^{m / 2-j} n_{\varepsilon}\right) \partial_{k}\left(M(-s) \partial_{s}^{j} E_{\varepsilon}\right)\right\|_{L^{2}} \\
& +\left\|\left(\partial_{s}^{m / 2-j} n_{\mathfrak{q}}\right) \partial_{k}^{2}\left(M(-s) \partial_{s}^{j} E_{\mathbb{q}}\right)\right\|_{\left.L^{2}\right] d s} \\
& \leqq C+C \int_{0}^{t} s^{2} \sum_{j=0}^{m / 2-1}\left[\left\|\partial_{k}^{2} \partial_{s}^{m / 2-j} n_{\mathrm{q}}\right\|_{L^{2}}\left\|\partial_{s}^{j} E_{\mathrm{q}}\right\|_{L^{\infty}}\right. \\
& +\left\|\partial_{k} \partial_{s}^{m / 2-j} n_{\mathrm{g}}\right\|_{L^{4}}\left\|\partial_{k}\left(M(-s) \partial_{s}^{j} E\right)\right\|_{L^{4}} \\
& +\left\|\partial_{s}^{m / 2-j} n_{\mathrm{q}}\right\|_{L^{\infty}}\left\|\partial_{k}^{2}\left(M(-s) \partial_{s}^{j} E_{\varepsilon}\right)\right\|_{\left.L^{2}\right] d s} \\
& +C \int_{0}^{t} s^{2}\left\|\partial_{k}^{2} n_{\mathrm{\varepsilon}}\right\|_{L^{\infty}}\left\|\partial_{s}^{m / 2} E_{\mathrm{g}}\right\|_{L^{2}} d s \\
& +C \int_{0}^{t} s^{2}\left\|\partial_{k} n_{\mathrm{g}}\right\|_{L^{\infty}}\left\|\partial_{k}\left(M(-s) \partial_{s}^{m / 2} E_{\mathrm{\varepsilon}}\right)\right\|_{L^{2}} d s \\
& +C \int_{0}^{t} s^{2}\left\|n_{\mathrm{g}}\right\|_{L^{\infty}}\left\|\partial_{k}^{2}\left(M(-s) \partial_{s}^{m / 2} E_{\mathrm{\varepsilon}}\right)\right\|_{L^{2}} d s, \\
& 1 \leqq k \leqq N \text {. }
\end{aligned}
$$

On the other hand, by the Sobolev imbedding theorem and the interpolation inequality we have

$$
\begin{aligned}
& \left\|\partial_{k}\left(M(-s) \partial_{s}^{j} E_{\mathrm{q}}\right)\right\|_{L^{4}} \\
& \quad \leqq C\left[\sum_{l=1}^{N}\left\|\partial_{l}^{2}\left(M(-s) \partial_{s}^{j} E_{s}\right)\right\|_{L^{2}}+\left\|\partial_{k}\left(M(-s) \partial_{s}^{j} E_{\mathrm{\varepsilon}}\right)\right\|_{L^{2}}\right] \\
& \left\|\partial_{k}\left(M(-s) \partial_{s}^{j} E_{\mathrm{\varepsilon}}\right)\right\|_{L^{2}} \\
& \quad \leqq C\left[\sum_{l=1}^{N}\left\|\partial_{l}^{2}\left(M(-s) \partial_{s}^{j} E_{\mathrm{\varepsilon}}\right)\right\|_{L^{2}}+\left\|\partial_{s}^{j} E_{\mathrm{q}}\right\|_{L^{2}}\right] .
\end{aligned}
$$

(4.43)-(4.45), (4.15), (4.38), (4.39) and the Sobolev imbedding theorem give us

$$
\begin{aligned}
& \sum_{k=1}^{N}\left\|J_{k}^{2} \partial_{\varepsilon}^{m / 2} E_{\varepsilon}(t)\right\|_{L^{2}} \leqq C \\
& +C \int_{0}^{t} s^{2} \sum_{j=0}^{m / 2-1}\left[\left\|\partial_{s}^{m / 2-j} n_{\mathrm{q}}\right\|_{H^{2}}\left\|\partial_{s}^{j} E_{\mathrm{q}}\right\|_{H^{2}}\right. \\
& +\left\|\partial_{s}^{m / 2-j} n_{\mathrm{q}}\right\|_{H^{2}}\left\{\sum_{k=1}^{N}\left\|\partial_{k}^{2}\left(M(-s) \partial_{s}^{j} E_{\mathrm{q}}\right)\right\|_{L^{2}}\right. \\
& \left.+\sum_{k=1}^{N}\left\|\partial_{k}\left(M(-s) \partial_{s}^{j} E_{\mathrm{\varepsilon}}\right)\right\|_{\left.L^{2}\right\}}\right] d s \\
& +C \int_{0}^{t} s^{2}\left\|n_{\mathrm{\varepsilon}}\right\|_{H^{4}}\left\|\partial_{s}^{m / 2} E_{\mathrm{\varepsilon}}\right\|_{L^{2}} d s
\end{aligned}
$$




$$
\begin{gathered}
+C \int_{0}^{t} s^{2}\left\|n_{\mathrm{\varepsilon}}\right\|_{H^{3}} \sum_{k=1}^{N}\left\|\partial_{k}\left(M(-s) \partial_{s}^{m / 2} E_{\mathrm{\varepsilon}}\right)\right\|_{L^{2}} d s \\
+C \int_{0}^{t} s^{2}\left\|n_{\mathrm{q}}\right\|_{H^{2}} \sum_{k=1}^{N}\left\|\partial_{k}^{2}\left(M(-s) \partial_{s}^{m / 2} E_{\mathrm{\varepsilon}}\right)\right\|_{L^{2}} d s \\
\leqq C_{12}+C_{13} \int_{0}^{t}(2 s)^{2} \sum_{k=1}^{N}\left\|\partial_{k}^{2}\left(M(-s) \partial_{s}^{m / 2} E_{\mathrm{\varepsilon}}\right)\right\|_{L^{2}} d s \\
=C_{12}+C_{13} \int_{0}^{t} \sum_{k=1}^{N}\left\|J_{k}^{2} \partial_{s}^{m / 2} E_{\mathrm{\varepsilon}}\right\|_{L^{2}} d s, \\
0 \leqq t \leqq T,
\end{gathered}
$$

where $C_{12}$ and $C_{13}$ depend only on $T,\left\|E_{0}\right\|_{H^{m, 2}, A(n)}$ and $N$ but not on $\varepsilon$. (4.46) and Gronwall's inequality yield

$$
\begin{aligned}
& 4 t^{2} \sum_{k=1}^{N} \| \partial_{k}^{2}(\left.M(-t) \partial_{t}^{m / 2} E_{\mathrm{g}}(t)\right) \|_{L^{2}} \\
&=\sum_{k=1}^{N}\left\|J_{k}^{2} \partial_{t}^{m / 2} E_{\mathrm{g}}(t)\right\|_{L^{2}} \leqq C_{14}, \quad 0<t \leqq T,
\end{aligned}
$$

where $C_{14}$ depends only on $T,\left\|E_{0}\right\|_{H^{m, 2}}, A(n)$ and $N$ but not on $\varepsilon$.

We can easily see that

$$
E_{\mathrm{\varepsilon}}(t) \rightarrow E(t) \quad i n \bigcap_{j=0}^{m / 2} C^{j}\left([0, T] ; H^{m-2 j}\right)
$$

as $\varepsilon \rightarrow 0$. Therefore, (4.47) and (4.48) imply that

$$
4 t^{2} \sum_{k=1}^{N}\left\|\partial_{k}^{2}\left(M(-t) \partial_{t}^{m / 2} E(t)\right)\right\|_{L^{2}} \leqq C_{14}, \quad 0<t \leqq T .
$$

(4.49) and the definition of $M(t)$ show that

$$
\begin{aligned}
\partial_{\varepsilon}^{m / 2} E \in L^{\infty}\left(\tau, T ; H^{2}(|x|<R)\right), \\
0<\tau<T, \quad R>0 .
\end{aligned}
$$

We differentiate (1.1) $m / 2-1$ times in $t$ to obtain

$$
\begin{aligned}
i \partial_{t}^{m / 2} E & +\Delta\left(\partial_{t}^{m / 2-1} E\right) \\
& =\sum_{j=0}^{m / 2-1}\left(\begin{array}{c}
m / 2-1 \\
j
\end{array}\right)\left(\partial_{t}^{m / 2-j} n\right)\left(\partial_{t}^{j} E\right), \quad 0 \leqq t \leqq T .
\end{aligned}
$$

Since $(E(t), n(t))$ is in the class of Theorem 1.1(2), the Sobolev imbedding theorem implies that the right hand side of (4.51) belongs to $L^{\infty}\left(0, T ; H^{2}\right)$. Therefore, (4.50), (4.51) and the local regularity theorem of the elliptic equation give us 


$$
\begin{aligned}
& \partial_{t}^{m / 2-1} E \in L^{\infty}(\tau, T ;\left.H^{4}(|x|<R)\right), \\
& 0<\tau<T, \quad R>0 .
\end{aligned}
$$

Repeating the above argument, we conclude that

$$
\begin{aligned}
& E \in L^{\circ}\left(\tau, T ; H^{m+2}(|x|<R)\right), \\
& 0<\tau<T, \quad R>0 .
\end{aligned}
$$

Thus, since $T$ is an arbitrary constant with $0<T<T_{\text {max }}$, the proof is completed in the case where $m$ is an even integer with $m \geqq 6$ and $k=2$.

In the same way as above we can prove Theorem 1.2(2) for the other cases. 閶

\section{References}

[1] Added, H. and Added, S., Existence globale de solutions fortes pour les équations de la turbulence de Langmuir en dimension 2, C.R. Acad. Sci. Paris, 299 (1984), 551554.

[2] —- Equations of Langmuir turbulence and nonlinear Schrödinger equation: Smoothness and approximation, J. Funct. Anal., 79 (1988), 183-210.

[ 3 ] Brezis, H. and Gallouet, T., Non linear Schrödinger evolution equations, Nonlinear Analysis, TMA, 4 (1980), 677-681.

[4] Constantin, P. and Saut, J.C., Local smoothing properties of dispersive equations, J. Amer. Math. Soc., 且 (1988), 413-439.

[ 5 ] Ginibre, J. and Velo, G., On a class of nonlinear Schrödinger equations I: the Cauchy problem, J. Funct. Anal., 32 (1979), 1-32.

[6] - Scattering theory in the energy space for a class of nonlinear Schrödinger equations, J. Math. Pures Appl., 64 (1985), 363-401.

[7] Hayashi, N., Nakamitsu, K. and Tsutsumi, M., On solutions of the initial value problem for the nonlinear Schrödinger equations in one space dimension, Math. Z., 192 (1986), 637-650.

[ 8 ] - On solutions of the initial value problem for the nonlinear Schrödinger equations, J. Funct. Anal., 71 (1987), 218-243.

[9] Hayashi, N. and Ozawa, T., Smoothing effect for some Schrödinger equations, J. Funct. Anal., 85 (1989), 307-348.

[10] Jensen, A., Commutator methods and a smoothing property of the Schrödinger evolution group, Math. Z., 191 (1986), 53-59.

[11] Kato, T., On nonlinear Schrödinger equations, Ann Inst. Henri Poincaré, Phys. Théor., 46 (1987), 113-129.

[12] Schochet, S.H. and Weinsteir, M.I., The nonlinear Schrödinger limit of the Zakharov equations governing Langmuir turbulence, Comm. Math. Phys., 106 (1986), 569-580.

[13] Shibata, Y. and Tsutsumi, Y., Local existence of solutions for the initial boundary value problem of fully nonlinear wave equation, Nonlinear Analysis, TMA, $\mathbb{1}$ (1987), 335-365.

[14] Sjölin, P., Regularity of solutions to the Schrödinger equation, Duke Math. J., 55 (1987), 699-715.

[15] - Local regularity of solutions to nonlinear Schrödinger equations, Ark. Mat., 28 (1990), 145-157.

[16] Strichartz, R.S., Restrictions of Fourier transforms to quadratic surfaces and decay of 
solutions of wave equations, Duke Math. J., 44 (1977), 705-714.

[17] Sulem, C. and Sulem, P.L., Quelques résultats de régularité pour les équations de la turbulence de Langmuir, C.R. Acad. Sci. Paris, 289 (1979), 173-176.

[18] Weinstein, M., Nonlinear Schrödinger equations and sharp interpolation estimates, Comm. Math. Phys., 87 (1983), 567-576.

[19] Yajima, K., Existence of solutions for Schrödinger evolution equations, Comm. Math. Phys., 110 (1987), 415-426.

[20] Zakharov, V.E., Collapse of Langmuir waves, Sov, Phys. JETP, 35 (1972), 908-914. 
